\title{
DECAY AND SCATTERING IN ENERGY SPACE FOR THE SOLUTION OF WEAKLY COUPLED SCHRÖDINGER-CHOQUARD AND HARTREE-FOCK EQUATIONS
}

\author{
M. TARULLI AND G. VENKOV
}

\begin{abstract}
We prove decay with respect to some Lebesgue norms for a class of Schrödinger equations with non-local nonlinearities by showing new Morawetz inequalities and estimates. As a byproduct, we obtain large-data scattering in the energy space for the solutions to the systems of $N$ defocusing Schrödinger-Choquard equations with massenergy intercritical nonlinearities in any space dimension and of defocusing Hartree-Fock equations, for any dimension $d \geqslant 3$.
\end{abstract}

\section{INTRODUCTION}

The primary target of the paper is the study of the decaying and scattering properties of the solution to the following system of $N \geqslant 1$ nonlinear evolution equations in dimension $d \geqslant 1$ :

$$
\left\{\begin{array}{l}
i \partial_{t} \psi_{j}+\Delta \psi_{j}-\sum_{k=1}^{N} G\left(\psi_{j}, \psi_{k}\right)=0 \\
\left(\psi_{j}(0, \cdot)\right)_{j=1}^{N}=\left(\psi_{j, 0}\right)_{j=1}^{N} \in H^{1}\left(\mathbb{R}^{d}\right)^{N}
\end{array}\right.
$$

characterized by the nonlinearities

$$
\begin{array}{r}
G\left(\psi_{j}, \psi_{k}\right)=\lambda_{j k}\left[|x|^{-\left(d-\gamma_{1}\right)} *\left|\psi_{k}\right|^{p}\right]\left|\psi_{j}\right|^{p-2} \psi_{j} \\
+\beta\left(\left[|x|^{-\left(d-\gamma_{2}\right)} *\left|\psi_{k}\right|^{2}\right] \psi_{j}-\left[|x|^{-\left(d-\gamma_{2}\right)} * \bar{\psi}_{k} \psi_{j}\right] \psi_{k}\right) .
\end{array}
$$

Here, for all $j, k=1, \ldots, N, \psi_{j}=\psi_{j}(t, x): \mathbb{R} \times \mathbb{R}^{d} \rightarrow \mathbb{C},\left(\psi_{j}\right)_{j=1}^{N}=$ $\left(\psi_{1}, \ldots, \psi_{N}\right)$ and $\beta, \lambda_{j k} \geqslant 0$ are coupling parameters such that $\lambda_{j j} \neq 0$, $\beta=0$ if $p>2$. Henceforth, we name (1.1) as Schrödinger-Choquard (SCH) if $p \geqslant 2$ and Hartree-Fock (HF) if $\lambda_{j k}=0$ for all $j, k=1, \ldots, N, N \geqslant 2$ in (1.2). We will require that the nonlinearity parameters $p, \gamma_{1}$ satisfy the

2010 Mathematics Subject Classification. 35J10, 35Q55, 35P25.

Key words and phrases. Nonlinear Schrödinger systems, Choquard equation, HartreeFock equations, scattering theory, weakly coupled equations. 
following relations

$$
\begin{gathered}
0<\gamma_{1}<d, \quad 2 \leqslant p<p^{*}(d), \quad p^{*}(d)= \begin{cases}\infty & \text { if } d=1,2, \\
\frac{d+\gamma_{1}}{d-2} & \text { if } d \geqslant 3,\end{cases} \\
p>p_{*}(d), \quad p_{*}(d)=\frac{d+\gamma_{1}+2}{d}
\end{gathered}
$$

that is the $L^{2}$-supercritical and $H^{1}$-subcritical regime. We shall assume also that $\max (0, d-4)<\gamma_{2}<d$ if $\beta \neq 0$. The system (1.1) enjoys two important conserved quantities: we have the mass

$$
M\left(\psi_{j}\right)(t)=\int_{\mathbb{R}^{d}}\left|\psi_{j}(t)\right|^{2} d x
$$

for any $j=1, \ldots, N$ and the energy,

$$
\begin{aligned}
& E\left(\psi_{1}, \ldots, \psi_{N}\right)=\sum_{j=1}^{N} \int_{\mathbb{R}^{d}}\left|\nabla \psi_{j}\right|^{2}+\frac{1}{2 p} \sum_{j, k=1}^{N} \lambda_{j k} \int\left(|x|^{-\left(n-\gamma_{1}\right)} *\left|\psi_{k}\right|^{p}\right)\left|\psi_{j}\right|^{p} d x \\
& \quad+\frac{\beta}{2} \sum_{j, k=1}^{N} \int_{\mathbb{R}^{d}}\left(\left[|x|^{-\left(d-\gamma_{2}\right)} *\left|\psi_{k}\right|^{2}\right]\left|\psi_{j}\right|^{2}-\left[|x|^{-\left(d-\gamma_{2}\right)} * \bar{\psi}_{k} \psi_{j}\right] \psi_{k} \bar{\psi}_{j}\right) d x .
\end{aligned}
$$

The equation (1.1) has a strong physical meaning and its role is important in many models of mathematical physics. In fact, the special case of the Hartree-Newton equation, that is when $d=3, p=2, N=1, \gamma_{1}=2$ and $\beta=0$ in (1.2), was variously introduced in the scenario of quantum mechanics in order to represent the mean-field limit of large systems of bosons (the so-called Bose-Einstein condensates) by considering the selfinteractions of the such charged particles. We suggest, in this direction, [12], [22], [26] and the references therein. About the HF equation, that is the case when $d=3, N \geqslant 2$ and $\lambda_{j k}=0, j, k=1 \ldots, N$ in (1.2), it was applied in [14] for certain approximations in the theory of one component, for portraying an exchange term resulting from Pauli's principle as well as for describing the fermions as an approximation of the equation overlooking the impact of their fermionic nature. Other relevant papers about this topic are [4] and [5] (see also the references inside). Furthermore, in [15] the Hartree-Fock equation was fundamental for developing models of white dwarfs. Turning to the SCH equation, the case of $d=3, \beta=0, p, \gamma_{1}$ as in (1.3) and $N=1$ in (1.1) was introduced to sketch an electron trapped in its own hole, as showed in [10] and [11] and very recently in [35], to describe self-gravitating matter together with quantum entanglement and quantum information effects. Morivated by this and by [8], where the general case of systems of interacting particles is studied, we carry on with the analysis of the decay properties of the solution to (1.1) unfolding large-data scattering in $H^{1}\left(\mathbb{R}^{d}\right)^{N}$ for the Schrödinger-Choquard and the Hartree-Fock systems 
on $N$ particles. By pursuing the ideas initially introduced in [7] for systems of Nonlinear Schrödinger equations with local nonlinearities (see also [39], [40] for the single NLS and [37] for the fourth-order NLS), we introduce relevant breakthroughs extending the theory to the non-local setting. Namely, the system (1.1) is translation invariant so we can set up either the Morawetz viriel and action, or their bilinear analogues. As a main outcome, we are able to present new Morawetz identities, interaction Morawetz identities and their associated inequalities for (1.1). The succeeding step is to localize the Morawetz inequalities on space-time slabs having $\mathbb{R}^{d}$-cubes as space components, utilizing again the translation invariance of the equation and of all the estimates involved. We say, that at level of localized frame, the dichotomy between local and non-local interactions breaks down: the convolution functions appearing in the interaction Morawetz can be handled in the same manner as if we are treating pure power nonlinearities. The corresponding localized estimates accomplish a contradiction argument which implies the decay of $L^{r}$-norms of the solutions $\left(\psi_{j}(t, x)\right)_{j=1}^{N}$, provided that $2<r \leqslant 2 d /(d-2)$, for $d \geqslant 3$ and, if $p>2,2<r<\infty$ for $d=1$, 2, with $r=\infty$ included for $d=1$. Let us underline that our approach guarantees the possibility to deal with the SCH in low spatial dimension $d=1,2$, bypassing the techniques of [33]. Now, this peculiar behaviour, jointly with a suitable reformulation of the theory developed in [9], bears to the asymptotic completeness and existence of the wave operators in the energy space $H^{1}\left(\mathbb{R}^{d}\right)^{N}$ for solution to (1.1). We point out now the novelties introduced in our paper. Looking at the Schrödinger-Hartree equation (that is, SCH with $p=2$ ) and at the HF systems in dimension $d \geqslant 3$, one knows that the aforementioned decay and the consequent scattering are similarly achieved in several papers like [18], [19], [34], [41], where the pseudo-conformal technique was successfully applied once one assumes the initial data laying in a weighted energy space. We improve all these results by selecting the initial data in $H^{1}\left(\mathbb{R}^{d}\right)^{N}$ only, showing a similar decay of the solution to (1.1) in the range $\max (0, d-4)<\gamma_{1}, \gamma_{2}<d$. We refine also the decay property of the solutions and simplify some of the results released in [20] and [21], where the scattering in the energy space for the Schrödinger-Hartree equation is acquired, for $\max (0, d-4)<\gamma_{1}<d-2$, without imposing further regularity to the initial data. Let us move to the case of the defocusing SCH given by (1.1) with $d \geqslant 1, N \geqslant 1, p>2$ in (1.2). We earn in this setting the full decay of the solution of the system (1.1), the existence of the scattering states and that the wave operators are well-defined and bijective in the energy-space $H^{1}\left(\mathbb{R}^{d}\right)$. Moreover, all such properties are transposed to the special case of $N=1$, that is

$$
\left\{\begin{array}{l}
i \partial_{t} \psi+\Delta \psi=\lambda\left(|x|^{-\left(n-\gamma_{1}\right)} *|\psi|^{p}\right)|\psi|^{p-2} \psi, \\
\psi(0, x)=\psi_{0}(x),
\end{array}\right.
$$

with $\lambda>0$. Currently, we are unaware of alike results, so we emphasize that ours are new in the whole literature. This explains the reason why we can 
not supply any kind of references.

The first main target of this paper is the following.

Theorem 1.1. Let $\left(\psi_{j}\right)_{j=1}^{N} \in \mathcal{C}\left(\mathbb{R}, H^{1}\left(\mathbb{R}^{d}\right)^{N}\right)$ be the unique global solution to (1.1) with $p>2$, and $\gamma_{1}$ in (1.2) such that (1.3) holds. Then, for all $j=1, \ldots, N$, one has the decay property

$$
\lim _{t \rightarrow \pm \infty}\left\|\psi_{j}(t)\right\|_{L^{r}\left(\mathbb{R}^{d}\right)}=0
$$

with $2<r \leqslant 2 d /(d-2)$, for $d \geqslant 3$, with $2<r<+\infty$, for $d=2$ and with $2<r \leqslant+\infty$, for $d=1$. Let $d \geqslant 3$, if $\left(\psi_{j}\right)_{j=1}^{N} \in \mathcal{C}\left(\mathbb{R}, H^{1}\left(\mathbb{R}^{d}\right)^{N}\right)$ is the unique global solution to (1.1) with $p=2$ and $\max (0, d-4)<\gamma_{1}, \gamma_{2}<d$ in (1.2), then (1.8) remains valid along with $2<r \leqslant 2 d /(d-2)$.

The second main result concerns the scattering of the solution in the energy space.

Theorem 1.2. Assume $d \geqslant 1$ and $p>2, \gamma_{1}$ such that (1.3), (1.4) hold or $d \geqslant 3$ and $p=2, \max (0, d-4)<\gamma_{1}, \gamma_{2}<d-2$, in (1.2). Let $\left(\psi_{j}\right)_{j=1}^{N} \in$ $\mathcal{C}\left(\mathbb{R}, H^{1}\left(\mathbb{R}^{d}\right)^{N}\right)$ be the unique global solution to (1.1), then:

- (asymptotic completeness) There exists $\left(\psi_{j, 0}^{ \pm}\right)_{j=1}^{N} \in H^{1}\left(\mathbb{R}^{d}\right)^{N}$ such that for all $j=1, \ldots, N$

$$
\lim _{t \rightarrow \pm \infty}\left\|\psi_{j}(t, \cdot)-e^{i t \Delta} \psi_{j, 0}^{ \pm}(\cdot)\right\|_{H^{1}\left(\mathbb{R}^{d}\right)}=0 .
$$

- (existence of wave operators) For every $\left(\psi_{j, 0}^{ \pm}\right)_{j=1}^{N} \in H^{1}\left(\mathbb{R}^{d}\right)^{N}$ there exists unique initial data $\left(\psi_{j, 0}\right)_{j=1}^{N} \in H^{1}\left(\mathbb{R}^{d}\right)^{N}$, such that the global solution to (1.1) $\left(\psi_{j}\right)_{j=1}^{N} \in \mathcal{C}\left(\mathbb{R}, H^{1}\left(\mathbb{R}^{d}\right)^{N}\right)$ satisfies (1.9).

Remark 1.3. We observe that (1.3) and (1.4) force to some restrictions on $\gamma_{1}$ above. As long as $p>2$, we need only that $p^{*}(d)>2$, for $d \geqslant 3$. This is equivalent to the condition $\gamma_{1}>\max (0, d-4)$. In dimensions $d=1,2$ the fact that $p_{*}(d)>2$ and $p^{*}(d)=\infty$, grants the full range $0<\gamma_{1}<d$. Unlike above, if $p=2$ then one has to require $p_{*}(d)<2<p^{*}(d)$. This compels to the conditions $\max (0, d-4)<\gamma_{1}, \gamma_{2}<d-2$, which are mandatory for the well-posedness and the asymptotic completness.

Then, Theorem 1.2 leads directly to other consequences. First we have the immediate one for the $\mathrm{SCH}$ equation:

Corollary 1.4. Let $d \geqslant 1$ and $p, \gamma_{1}$ as in (1.3). Then, if $\psi_{0} \in H^{1}\left(\mathbb{R}^{d}\right)$, the unique global solution $\psi \in \mathcal{C}\left(\mathbb{R}, H^{1}\left(\mathbb{R}^{d}\right)\right)$ to $(1.7)$ is such that:

- if $p>2$, the decay property

$$
\lim _{t \rightarrow \pm \infty}\|\psi(t, \cdot)\|_{L^{r}\left(\mathbb{R}^{d}\right)}=0
$$

is verified for $2<r \leqslant 2 d /(d-2), d \geqslant 3$, for $2<r<+\infty, d=2$ and for $2<r \leqslant+\infty, d=1$; 
- if $p>\max \left(2, p_{*}(d)\right)$, the scattering occurs, i.e. there exists $\psi_{0}^{ \pm} \in$ $H^{1}\left(\mathbb{R}^{d}\right)$ such that

$$
\lim _{t \rightarrow \pm \infty}\left\|\psi(t, \cdot)-e^{-i t \Delta} \psi_{0}^{ \pm}(\cdot)\right\|_{H^{1}\left(\mathbb{R}^{d}\right)}=0 .
$$

In the Schrödinger-Hartree and HF systems framework we have:

Corollary 1.5. Let $d \geqslant 3, p=2$ and $\max (0, d-4)<\gamma_{1}, \gamma_{2}<d-2$ in (1.2). Then, if $\left(\psi_{j, 0}^{ \pm}\right)_{j=1}^{N} \in H^{1}\left(\mathbb{R}^{d}\right)^{N}$, the unique global solution $\left(\psi_{j}\right)_{j=1}^{N} \in$ $\mathcal{C}\left(\mathbb{R}, H^{1}\left(\mathbb{R}^{d}\right)^{N}\right)$ to $(1.1)$ is such that:

- the decay property

$$
\lim _{t \rightarrow \pm \infty}\left\|\psi_{j}(t, \cdot)\right\|_{L^{r}\left(\mathbb{R}^{d}\right)}=0
$$

is fulfilled for $2<r \leqslant 2 d /(d-2)$;

- the scattering occurs, i.e. there exists $\left(\psi_{j, 0}^{ \pm}\right)_{j=1}^{N} \in H^{1}\left(\mathbb{R}^{d}\right)^{N}$ such that

$$
\lim _{t \rightarrow \pm \infty}\left\|\psi_{j}(t, \cdot)-e^{i t \Delta} \psi_{j, 0}^{ \pm}(\cdot)\right\|_{H^{1}\left(\mathbb{R}^{d}\right)}=0 .
$$

Remark 1.6. The foregoing corollary summarizes different results. In the case $p=2$ and $\beta=0$, we get (1.12) displaced for the system of $N \geqslant 1$ coupled Schrödinger-Hartree equations and if $N \geqslant 2$ and $\lambda_{j k}=0$ for all $j, k=1 \ldots, N$, we have the same decay property for the solution of the HF equation. Once (1.12) is proved, we can construct the scattering operators in the energy space.

The literature related to these subjects is not so wide and according to our knowledge, Morawetz and interaction Morawetz estimates were available for systems of NLS for the first time in [7] and successively in [37]. We come to an end by itemizing briefly some other achievements, different from the already cited ones, which regard particular versions of (1.1). The wellposedness for the Schrödinger-Hartree equation, both local and global, was examined in [9], [24] and [30] while the existence of the standing waves was discussed in [27]. The scattering in the focusing critical case was examined in [29] and the blow up of the solutions in the focusing framework, in [31] (we suggest the references contained therein also). As we said, little is known for the single SCH. On the other hand we cite here [13] for the well-posedness for the single $\mathrm{SCH}$ and [27] for the well-posedness and blow-up in the case of SCH perturbed by an inverse square potential. We remind [16], in which local and global well-posedness, existence of standing waves and blow up solutions were investigated for (1.7) with $\gamma_{1}=2$ for $p=(d+4) / d$ in the focusing case $\lambda<0$. We mention also [6], [17] and [32], for more general informations about the solitary waves solution of the focusing (1.7). In closing, we recall that scattering for the focusing SCH in $d \geqslant 3$, was earned in [2] and in [3] for large radial data and small data, respectively. 
Outline of paper. After some preliminaries in Section 2, through the Section 3 we build, in Lemma 3.1 and Lemma 3.2, the Morawetz inequalities and their bilinear counterpart, respectively. The principal target of the Section 4 is to unveil the decay of some Lebesgue norms of the solutions to the systems (1.1), which is a fundamental property for catching the scattering states and is included in Proposition 1.1. Finally, all the remaining scattering theory associated to (1.1) takes place in Section 5. The last section is the Appendix A, in which a localized Gagliardo-Nirenberg inequality, an ancillary tool used extensively beside the paper, is obtained.

\section{Preliminaries}

We indicate by $L_{x}^{r}$ the Lebesgue space $L^{r}\left(\mathbb{R}^{d}\right)$, and by $W_{x}^{1, r}$ and $H_{x}^{1}$ the inhomogeneous Sobolev spaces $W^{1, r}\left(\mathbb{R}^{n}\right)$ and $H^{1}\left(\mathbb{R}^{n}\right)$, respectively (for more details see [1]). For any $N \in \mathbb{N}$, we also define $\mathcal{L}_{x}^{r}=L^{r}\left(\mathbb{R}^{d}\right)^{N}$ and introduce the Sobolev spaces $\mathcal{W}_{x}^{1, r}=W^{1, r}\left(\mathbb{R}^{d}\right)^{N}$ and $\mathcal{H}_{x}^{1}=H^{1}\left(\mathbb{R}^{d}\right)^{N}$. From now on and in the sequel we adopt the following notations: for any two positive real numbers $a, b$, we write $a \lesssim b$ (resp. $a \gtrsim b)$ to denote $a \leqslant C b($ resp. $C a \geqslant b)$, with $C>0$, we unfold the constant only when it is essential. We recall also some of the results concerning the well-posedness for (1.1) already available, such as [3] [13], [27] for the SCH and as [21], [34], [41] in the HF framework. Then we can summarize them as:

Proposition 2.1. Let $d \geqslant 1$ and assume (1.2) is such that $p>2, \gamma_{1}$ satisfy (1.3) or $p=2, \max (0, d-4)<\gamma_{1}, \gamma_{2}<d$. Then for all $\left(\psi_{j, 0}\right)_{j=1}^{N} \in \mathcal{H}_{x}^{1}$ there exists a unique global solution $\left(\psi_{j}\right)_{j=1}^{N} \in \mathcal{C}\left(\mathbb{R}, \mathcal{H}_{x}^{1}\right)$ to (1.1), moreover

$$
M\left(\psi_{j}\right)(t)=\left\|\psi_{j}(0)\right\|_{L_{x}^{2}}
$$

for all $j=1, \ldots, N$ and

$$
E\left(\psi_{1}(t), \ldots, \psi_{N}(t)\right)=E\left(\psi_{1}(0), \ldots, \psi_{N}(0)\right),
$$

with $E\left(\psi_{1}(t), \ldots, \psi_{N}(t)\right)$ as in (1.6).

The proposition above can be obtained by standard energy method (see Theorem 3.3.9 and Remark 3.3.12 in [9]) combined with the inequality

$$
\int_{\mathbb{R}^{d}}\left(|x|^{-(d-\gamma)} *|\psi|^{p}\right)|\psi|^{p} d x \lesssim\|\psi\|_{L_{x}^{\frac{2 p d}{d+\gamma}}}^{2 p} \lesssim\|\psi\|_{H_{x}^{1}}^{2 p},
$$

for $p \in[(d+\gamma) / d,(d+\gamma) /(d-2)](p \in[(d+\gamma) / d, \infty)$, if $d=1,2)$, as well as the defocusing nature of the system.

\section{Morawetz identities and nonlinear interaction Morawetz INEQUALITIES}

We provide, thorough this section, the fundamental tools for the proof of our first main theorem. We start by obtaining Morawetz-type identities, which are comparable to the ones holding for the single NLS. From now on we hide the variable $t$ for simplicity, spreading it out only when necessary. 
Moreover, we find suitable to set up the following notations: given a function $h \in H^{1}\left(\mathbb{R}^{d}, \mathbb{C}\right)$, we denote by

$$
m_{h}(x):=|h(x)|^{2}, \quad j_{h}(x):=\operatorname{Im}[\bar{h} \nabla h(x)],
$$

the mass and momentum densities, respectively. We have the Morawetz identities for non-local nonlinearities.

Lemma 3.1. Let $d \geqslant 1$ and $\left(\psi_{j}\right)_{j=1}^{N} \in \mathcal{C}\left(\mathbb{R}, H^{1}\left(\mathbb{R}^{d}\right)^{N}\right)$ be as in Proposition 2.1, let $a=a(x): \mathbb{R}^{d} \rightarrow \mathbb{R}$ be a sufficiently regular and decaying function, and indicate by

$$
\mathcal{V}(t):=\sum_{j=1}^{N} \int_{\mathbb{R}^{d}} a(x) m_{\psi_{j}}(x) d x .
$$

The following identities hold:

$$
\begin{array}{r}
\dot{\mathcal{V}}(t)=\sum_{j=1}^{N} \int_{\mathbb{R}^{d}} a(x) \dot{m}_{\psi_{j}}(x) d x=2 \sum_{j=1}^{N} \int_{\mathbb{R}^{d}} j_{\psi_{j}}(x) \cdot \nabla a(x) d x \\
\ddot{\mathcal{V}}(t)=\sum_{j=1}^{N} \int_{\mathbb{R}^{d}} a(x) \ddot{m}_{\psi_{j}}(x) d x
\end{array}
$$

$$
\begin{array}{r}
=\sum_{j=1}^{N}\left[-\int_{\mathbb{R}^{d}} m_{\psi_{j}(x)}(x) \Delta^{2} a(x) d x+4 \int_{\mathbb{R}^{d}} \nabla \psi_{j}(x) D^{2} a(x) \cdot \nabla \psi_{j}(x) d x\right] \\
+\frac{2(p-2)}{p} \sum_{j, k=1}^{N} \lambda_{j k} \int_{\mathbb{R}^{d}} \Delta a(x)\left[|x|^{-\left(d-\gamma_{1}\right)} *\left|\psi_{k}\right|^{p}\right]\left|\psi_{j}(x)\right|^{p} d x, \\
-\frac{4}{p} \sum_{j, k=1}^{N} \lambda_{j k} \int_{\mathbb{R}^{d}} \nabla a(x) \cdot \nabla\left[|x|^{-\left(d-\gamma_{1}\right)} *\left|\psi_{k}\right|^{p}\right]\left|\psi_{j}(x)\right|^{p} d x, \\
-2 \beta \sum_{j, k=1}^{N} \int_{\mathbb{R}^{d}} \nabla a(x) \cdot \nabla F\left(x, \psi_{j}, \bar{\psi}_{j}, \psi_{k}, \bar{\psi}_{k}\right) d x,
\end{array}
$$

with $\beta=0$ if $p>2$,

$$
\begin{array}{r}
F\left(x, \psi_{j}, \bar{\psi}_{j}, \psi_{k}, \bar{\psi}_{k}\right)= \\
{\left[|x|^{-\left(d-\gamma_{2}\right)} *\left|\psi_{k}\right|^{2}\right]\left|\psi_{j}(x)\right|^{2}-\left[|x|^{-\left(d-\gamma_{2}\right)} * \bar{\psi}_{k} \psi_{j}\right] \psi_{k}(x) \bar{\psi}_{j}(x),}
\end{array}
$$

for any $j, k=1, \ldots, N, D^{2} a \in \mathcal{M}_{d \times d}\left(\mathbb{R}^{d}\right)$ is the Hessian matrix of $a$ and $\Delta^{2} a=\Delta(\Delta a)$ the bi-laplacian operator.

Proof. We will proceed similarly to [7] (see also [37], [39]). We shall assume that $\left(\psi_{j}\right)_{j=1}^{N}$ is a smooth solution to (1.1), taking into account that the case $\left(\psi_{j}\right)_{j=1}^{N} \in \mathcal{C}\left(\mathbb{R}, H^{1}\left(\mathbb{R}^{d}\right)^{N}\right)$ can be established by a density argument (we cite, for example, [21]). The equation (3.2) is simple to derive. We carry out some 
details for providing (3.3) only. By means of an integration by parts and thanks to (1.1), we have

$$
\begin{array}{r}
2 \sum_{j=1}^{N} \partial_{t} \int_{\mathbb{R}^{d}} j_{\psi_{j}}(x) \cdot \nabla a(x) d x \\
=-2 \sum_{j=1}^{N} \operatorname{Im} \int_{\mathbb{R}^{d}} \partial_{t} \psi_{j}(x)\left[\Delta a(x) \bar{\psi}_{j}(x)+2 \nabla a(x) \cdot \nabla \bar{\psi}_{j}(x)\right] d x \\
=2 \sum_{j=1}^{N} \operatorname{Re} \int_{\mathbb{R}^{d}} i \partial_{t} \psi_{j}(x)\left[\Delta a(x) \bar{\psi}_{j}(x)+2 \nabla a(x) \cdot \nabla \bar{\psi}_{j}(x)\right] d x \\
=2 \sum_{j=1}^{N} \operatorname{Re} \int_{\mathbb{R}^{d}}\left[-\Delta \psi_{j}(x)+G\left(\psi_{j}, \psi_{k}\right)\right]\left[\Delta a(x) \bar{\psi}_{j}(x)+2 \nabla a(x) \cdot \nabla \bar{\psi}_{j}(x)\right] d x .
\end{array}
$$

First, one can get

$$
\begin{array}{r}
2 \sum_{j=1}^{N} \operatorname{Re} \int_{\mathbb{R}^{d}}-\Delta \psi_{j}(x)\left[\Delta a(x) \bar{\psi}_{j}(x)+2 \nabla a(x) \cdot \nabla \bar{\psi}_{j}(x)\right] d x \\
=-\sum_{j=1}^{N} \int_{\mathbb{R}^{d}} \Delta^{2} a(x)\left|\psi_{j}(x)\right|^{2} d x+4 \sum_{j=1}^{N} \int_{\mathbb{R}^{d}} \nabla \psi_{j}(x) D^{2} \psi_{j}(x) \nabla \bar{\psi}_{j}(x) d x .
\end{array}
$$

Furthermore we obtain

$$
\begin{aligned}
& 2 \sum_{j, k=1}^{N} \operatorname{Re} \int_{\mathbb{R}^{d}} G\left(\psi_{j}, \psi_{k}\right)\left[\Delta a(x) \bar{\psi}_{j}(x)+2 \nabla a(x) \cdot \nabla \bar{\psi}_{j}(x)\right] d x \\
= & 2 \sum_{j, k=1}^{N} \operatorname{Re} \int_{\mathbb{R}^{d}} G_{1}\left(\psi_{j}, \psi_{k}\right)\left[\Delta a(x) \bar{\psi}_{j}(x)+2 \nabla a(x) \cdot \nabla \bar{\psi}_{j}(x)\right] d x \\
+ & 2 \sum_{j, k=1}^{N} \operatorname{Re} \int_{\mathbb{R}^{d}} G_{2}\left(\psi_{j}, \psi_{k}\right)\left[\Delta a(x) \bar{\psi}_{j}(x)+2 \nabla a(x) \cdot \nabla \bar{\psi}_{j}(x)\right] d x,
\end{aligned}
$$


with

$$
\begin{array}{r}
2 \sum_{j, k=1}^{N} \operatorname{Re} \int_{\mathbb{R}^{d}} G_{1}\left(\psi_{j}, \psi_{k}\right)\left[\Delta a(x) \bar{\psi}_{j}(x)+2 \nabla a(x) \cdot \nabla \bar{\psi}_{j}(x)\right] d x \\
=2 \sum_{j, k=1}^{N} \lambda_{j k} \int_{\mathbb{R}^{d}} \Delta a(x)\left[|x|^{-\left(d-\gamma_{1}\right)} *\left|\psi_{k}\right|^{p}\right]\left|\psi_{j}(x)\right|^{p} d x \\
+4 \sum_{j, k=1}^{N} \lambda_{j k} \operatorname{Re} \int_{\mathbb{R}^{d}} \nabla a(x)\left[|x|^{-\left(d-\gamma_{1}\right)} *\left|\psi_{k}\right|^{p}\right] \cdot\left|\psi_{j}(x)\right|^{p-2} \psi_{j}(x) \nabla \bar{\psi}_{j}(x) d x \\
=2\left(1-\frac{2}{p}\right) \sum_{j, k=1}^{N} \lambda_{j k} \int_{\mathbb{R}^{d}} \Delta a(x)\left[|x|^{-\left(d-\gamma_{1}\right)} *\left|\psi_{k}\right|^{p}\right]\left|\psi_{j}(x)\right|^{p} d x \\
-\frac{4}{p} \sum_{j, k=1}^{N} \lambda_{j k} \int_{\mathbb{R}^{d}} \nabla a(x) \cdot \nabla\left[|x|^{-\left(d-\gamma_{1}\right)} *\left|\psi_{k}\right|^{p}\right]\left|\psi_{j}(x)\right|^{p} d x
\end{array}
$$

and

$$
\begin{array}{r}
2 \sum_{j, k=1}^{N} \operatorname{Re} \int_{\mathbb{R}^{d}} G_{2}\left(\psi_{j}, \psi_{k}\right)\left[\Delta a(x) \bar{\psi}_{j}(x)+2 \nabla a(x) \cdot \nabla \bar{\psi}_{j}(x)\right] d x \\
=2 \beta \sum_{j, k=1}^{N} \int_{\mathbb{R}^{d}} \Delta a(x)\left[|x|^{-\left(d-\gamma_{2}\right)} *\left|\psi_{k}\right|^{2}\right]\left|\psi_{j}(x)\right|^{2} d x \\
+2 \beta \sum_{j, k=1}^{N} \operatorname{Re} \int_{\mathbb{R}^{d}} 2 \nabla a(x)\left[|x|^{-\left(d-\gamma_{2}\right)} *\left|\psi_{k}\right|^{2}\right] \cdot \psi_{j}(x) \nabla \bar{\psi}_{j}(x) d x \\
-2 \beta \sum_{j, k=1}^{N} \int_{\mathbb{R}^{d}} \Delta a(x)\left[|x|^{-\left(d-\gamma_{2}\right)} * \bar{\psi}_{k} \psi_{j}\right] \psi_{k}(x) \bar{\psi}_{j}(x) d x \\
-2 \beta \sum_{j, k=1}^{N} \operatorname{Re} \int_{\mathbb{R}^{d}} 2 \nabla a(x)\left[|x|^{-\left(d-\gamma_{2}\right)} * \bar{\psi}_{k} \psi_{j}\right] \psi_{k}(x) \cdot \nabla \bar{\psi}_{j}(x) d x .
\end{array}
$$

An integration by parts of the the second term on the r.h.s. of the above identity (3.9) enhances to

$$
\begin{array}{r}
2 \beta \sum_{j, k=1}^{N} \operatorname{Re} \int_{\mathbb{R}^{d}} \nabla a(x)\left[|x|^{-\left(d-\gamma_{2}\right)} *\left|\psi_{k}\right|^{2}\right] \cdot \nabla\left|\psi_{j}(x)\right|^{2} d x \\
=-2 \beta \sum_{j, k=1}^{N} \int_{\mathbb{R}^{d}} \Delta a(x)\left[|x|^{-\left(d-\gamma_{2}\right)} *\left|\psi_{k}\right|^{2}\right]\left|\psi_{j}(x)\right|^{2} d x \\
-2 \beta \sum_{j, k=1}^{N} \int_{\mathbb{R}^{d}} \nabla a(x) \cdot \nabla\left[|x|^{-\left(d-\gamma_{2}\right)} *\left|\psi_{k}\right|^{2}\right]\left|\psi_{j}(x)\right|^{2} d x .
\end{array}
$$


By a further integration by parts, one has for the last term in (3.9), instead,

$$
\begin{array}{r}
4 \beta \sum_{j, k=1}^{N} \operatorname{Re} \int_{\mathbb{R}^{d}} \int_{\mathbb{R}^{d}} \nabla a(x) \frac{\bar{\psi}_{k}(y) \psi_{k}(x)}{|x-y|^{\left(d-\gamma_{2}\right)}} \cdot \nabla \psi_{j}(y) \bar{\psi}_{j}(x) d x d y \\
=-2 \beta \sum_{j, k=1}^{N} \int_{\mathbb{R}^{d}} \Delta a(x)\left[|x|^{-\left(d-\gamma_{2}\right)} * \bar{\psi}_{k} \psi_{j}\right] \psi_{k}(x) \bar{\psi}_{j}(x) d x \\
-2 \beta \sum_{j, k=1}^{N} \int_{\mathbb{R}^{d}} \nabla a(x) \cdot \nabla\left[|x|^{-\left(d-\gamma_{2}\right)} * \bar{\psi}_{k} \psi_{j}\right] \psi_{k}(x) \bar{\psi}_{j}(x) d x .
\end{array}
$$

We can utilize now (3.8) in combination with (3.9), (3.10) and (3.11) to rewrite (3.7) as

$$
\begin{array}{r}
2 \sum_{j=1}^{N} \operatorname{Re} \int_{\mathbb{R}^{d}} G\left(\psi_{j}, \psi_{k}\right)\left[\Delta a(x) \bar{\psi}_{j}(x)+2 \nabla a(x) \cdot \nabla \bar{\psi}_{j}(x)\right] d x \\
=\frac{2(p-2)}{p} \sum_{j, k=1}^{N} \lambda_{j k} \int_{\mathbb{R}^{d}} \Delta a(x)\left[|x|^{-\left(d-\gamma_{1}\right)} *\left|\psi_{k}\right|^{p}\right]\left|\psi_{j}(x)\right|^{p} d x, \\
-\frac{4}{p} \sum_{j, k=1}^{N} \lambda_{j k} \int_{\mathbb{R}^{d}} \nabla a(x) \cdot \nabla\left[|x|^{-\left(d-\gamma_{1}\right)} *\left|\psi_{k}\right|^{p}\right]\left|\psi_{j}(x)\right|^{p} d x, \\
-2 \beta \sum_{j, k=1}^{N} \int_{\mathbb{R}^{d}} \nabla a(x) \cdot \nabla F\left(x, \psi_{j}, \bar{\psi}_{j}, \psi_{k}, \bar{\psi}_{k}\right) d x,
\end{array}
$$

with $F\left(x, \psi_{j}, \bar{\psi}_{j}, \psi_{k}, \bar{\psi}_{k}\right)$ as in (3.4). Then the above identities (3.6) and (3.12) bring us to the proof of (3.2).

One can now apply the previous lemma for proving the following interaction Morawetz identities and inequalities for non-local nonlinearities.

Lemma 3.2. Let $\left(\psi_{j}\right)_{j=1}^{N} \in \mathcal{C}\left(\mathbb{R}, H^{1}\left(\mathbb{R}^{d}\right)^{N}\right)$ be as in Proposition 2.1, a= $a(|x|): \mathbb{R}^{d} \rightarrow \mathbb{R}$ be a convex radial, sufficiently regular and decaying function. Indicate by $a^{\star}=a^{\star}(x, y):=a(|x-y|): \mathbb{R}^{2 d} \rightarrow \mathbb{R}$ and by

$$
\mathcal{I}(t):=\sum_{j, \ell=1}^{N} \int_{\mathbb{R}^{d}} \int_{\mathbb{R}^{d}} a^{\star}(x, y) m_{\psi_{j}}(x) m_{\psi_{\ell}}(y) d x d y .
$$

The following holds:

$$
\begin{array}{r}
\dot{\mathcal{I}}(t)=2 \sum_{j, \ell=1}^{N} \int_{\mathbb{R}^{d}} \int_{\mathbb{R}^{d}} j_{\psi_{j}}(x) \cdot \nabla_{x} a^{\star}(x, y) m_{\psi_{\ell}}(y) d x d y, \\
N_{\left(p, a^{\star}\right)}^{C}(t)+N_{\left(2, a^{\star}\right)}^{H F}(t)+R_{\left(p, a^{\star}\right)}^{C}(t) \leqslant \ddot{\mathcal{I}}(t),
\end{array}
$$


where

$$
N_{\left(p, a^{\star}\right)}^{C}(t)=
$$

$$
\sum_{j, k, \ell=1}^{N} \widetilde{\lambda}_{j k} \int_{\mathbb{R}^{d}} \int_{\mathbb{R}^{d}} \Delta_{x} a^{\star}(x, y)\left[|x|^{-\left(d-\gamma_{1}\right)} *\left|\psi_{k}(x)\right|^{p}\right]\left|\psi_{j}(x)\right|^{p} m_{\psi_{\ell}}(y) d x d y,
$$

with $\widetilde{\lambda}_{j k}=4 \lambda_{j k}(p-2) / p$

$$
-\frac{8}{p} \sum_{j, k, \ell=1}^{N} \lambda_{j k} \int_{\mathbb{R}^{d}} \int_{\mathbb{R}^{d}} \nabla_{x} a^{\star}(x, y) \cdot \nabla_{x}\left[|x|^{-\left(d-\gamma_{1}\right)} *\left|\psi_{k}\right|^{p}\right]\left|\psi_{j}(x)\right|^{p} m_{\psi_{\ell}}(y) d x d y,
$$

$$
N_{\left(2, a^{\star}\right)}^{H F}(t)=
$$

$$
-4 \beta \sum_{j, k, \ell=1}^{N} \int_{\mathbb{R}^{d}} \int_{\mathbb{R}^{d}} \nabla_{x} a^{\star}(x, y) \cdot \nabla_{x} F\left(x, \psi_{j}, \bar{\psi}_{j}, \psi_{k}, \bar{\psi}_{k}\right) m_{\psi_{\ell}}(y) d x d y,
$$

with $\beta=0$ if $p>2$ and $F\left(x, \psi_{j}, \bar{\psi}_{j}, \psi_{k}, \bar{\psi}_{k}\right)$ as in (3.4).

Proof. As formerly done, we prove the identities for a smooth solution of (1.1), moving to the general case $\left(\psi_{j}\right)_{j=1}^{N} \in \mathcal{C}\left(\mathbb{R}, H^{1}\left(\mathbb{R}^{d}\right)^{N}\right)$ by an usual density argument. First, we point out that (3.13), because of the symmetry of the function $a^{\star}(x, y)=a(|x-y|)$, is equivalent to

$$
\dot{\mathcal{I}}(t)=2 \sum_{j, \ell=1}^{N} \int_{\mathbb{R}^{d}} \int_{\mathbb{R}^{d}} a^{\star}(x, y) \dot{m}_{\psi_{j}}(x) m_{\psi_{\ell}}(y) d x d y
$$

Hence, (3.13) is straightforward from (3.2) and Fubini's Theorem. We differentiate w.r.t. time variable working out now the equality

$$
\begin{array}{r}
=-4 \sum_{j, \ell=1}^{N} \operatorname{Re} \int_{\mathbb{R}^{d}} \int_{\mathbb{R}^{d}} m_{\psi_{\ell}}(y) i \partial_{t}\left(\bar{\psi}_{j}(x) \nabla_{x} \psi_{j}(x)\right) \cdot \nabla_{x} a^{\star}(x, y) d x d y \\
-2 \sum_{j, \ell=1}^{N} \operatorname{Re} \int_{\mathbb{R}^{d}} \int_{\mathbb{R}^{d}} i \partial_{t} m_{\psi_{j}}(x) \bar{\psi}_{\ell}(y) \nabla_{y} \psi_{\ell}(y) \cdot \nabla_{y} a^{\star}(x, y) d x d y \\
-2 \sum_{j, \ell=1}^{N} \operatorname{Re} \int_{\mathbb{R}^{d}} \int_{\mathbb{R}^{d}} i \partial_{t} m_{\psi_{\ell}}(y) \bar{\psi}_{j}(x) \nabla_{x} \psi_{j}(x) \cdot \nabla_{x} a^{\star}(x, y) d x d y \\
:=\mathcal{I I}_{1}(t)+\mathcal{I I}_{2}(t) .
\end{array}
$$


By the identity (3.3), Fubini's Theorem and the symmetry of $a^{\star}(x, y)$ we achieve

$$
\begin{array}{r}
\mathcal{I I}_{1}(t)=-2 \sum_{j, \ell=1}^{N} \int_{\mathbb{R}^{d}} \int_{\mathbb{R}^{d}} \Delta_{x}^{2} a^{\star}(x, y) m_{\psi_{j}}(x) m_{\psi_{\ell}}(y) d x d y \\
+\sum_{j, k, \ell=1}^{N} \widetilde{\lambda}_{j k} \int_{\mathbb{R}^{d}} \int_{\mathbb{R}^{d}} \Delta_{x} a^{\star}(x, y)\left[|x|^{-\left(d-\gamma_{1}\right)} *\left|\psi_{k}(x)\right|^{p}\right]\left|\psi_{j}(x)\right|^{p} m_{\psi_{\ell}}(y) d x d y,
\end{array}
$$$$
-\frac{8}{p} \sum_{j, k, \ell=1}^{N} \lambda_{j k} \int_{\mathbb{R}^{d}} \int_{\mathbb{R}^{d}} \nabla_{x} a^{\star}(x, y) \cdot \nabla_{x}\left[|x|^{-\left(d-\gamma_{1}\right)} *\left|\psi_{k}\right|^{p}\right]\left|\psi_{j}(x)\right|^{p} m_{\psi_{\ell}}(y) d x d y,
$$

$$
-4 \beta \sum_{j, k, \ell=1}^{N} \int_{\mathbb{R}^{d}} \int_{\mathbb{R}^{d}} \nabla_{x} a^{\star}(x, y) \nabla_{x} F\left(x, \psi_{j}, \bar{\psi}_{j}, \psi_{k}, \bar{\psi}_{k}\right) m_{\psi_{\ell}}(y) d x d y,
$$

The first term of (3.19) above arises from the linear part of the equation, while the other terms are connected to the nonlinearity contained in the equation. The linear term can be modified as follows

$$
\begin{aligned}
& -2 \sum_{j, \ell=1}^{N} \int_{\mathbb{R}^{d}} \int_{\mathbb{R}^{d}} \Delta_{x}^{2} a^{\star}(x, y) m_{\psi_{j}}(t, x) m_{\psi_{\ell}}(t, y) d x d y \\
= & 2 \sum_{i, j, \ell=1}^{N} \int_{\mathbb{R}^{d}} \int_{\mathbb{R}^{d}} \partial_{x_{i}} \partial_{y_{i}} \Delta_{x} a^{\star}(x, y) m_{\psi_{j}}(t, x) m_{\psi_{\ell}}(t, y) d x d y \\
= & 2 \sum_{j, \ell=1}^{N} \int_{\mathbb{R}^{d}} \int_{\mathbb{R}^{d}} \Delta_{x} a^{\star}(x, y) \nabla_{x} m_{\psi_{j}}(t, x) \cdot \nabla_{y} m_{\psi_{\ell}}(t, y) d x d y,
\end{aligned}
$$

by an integration by parts and taking again advantage of the property $\partial_{x_{i}} a^{\star}=-\partial_{y_{i}} a^{\star}$. At the end, we have

$$
\begin{array}{r}
\mathcal{I I}_{1}(t)=N_{\left(p, a^{\star}\right)}^{C}(t)+N_{\left(2, a^{\star}\right)}^{H F}(t)+R_{\left(p, a^{\star}\right)}^{C}(t) \\
+2 \sum_{j, \ell=1}^{N} \int_{\mathbb{R}^{d}} \int_{\mathbb{R}^{d}} \Delta_{x} a^{\star}(x, y) \nabla_{x} m_{\psi_{j}}(t, x) \cdot \nabla_{y} m_{\psi_{\ell}}(t, y) d x d y .
\end{array}
$$


As well, by the Morawetz identities (3.2), (3.3) and Fubini's Theorem we get

$$
\begin{aligned}
\mathcal{I I}_{2}(t)= & 4 \sum_{j, \ell=1}^{N} \int_{\mathbb{R}^{d}} \int_{\mathbb{R}^{d}} \nabla \psi_{j}(x) D_{x}^{2} a^{\star}(x, y) \nabla \bar{\psi}_{j}(x) m_{\psi_{\ell}}(y) d x d y \\
& +4 \sum_{j, \ell=1}^{N} \int_{\mathbb{R}^{d}} \int_{\mathbb{R}^{d}} m_{\psi_{j}}(x) \nabla \psi_{\ell}(y) D_{y}^{2} a^{\star}(x, y) \nabla \bar{\psi}_{\ell}(y) d x d y \\
& +8 \sum_{j, \ell=1}^{N} \int_{\mathbb{R}^{d}} \int_{\mathbb{R}^{d}} j_{\psi_{j}}(x) D_{x y}^{2} a^{\star}(x, y) \cdot j_{\psi_{\ell}}(y) d x d y
\end{aligned}
$$

here we applied, at this point, the symmetry of $D^{2} a^{\star}$ to drop the real part condition in the first two summands on the r.h.s. of the identity above. Once more, the fact that $\partial_{x_{i}} a^{\star}=-\partial_{y_{i}} a^{\star}$ allows us to reshape (3.22) as

$$
\begin{array}{r}
\mathcal{I I}_{2}(t)=4 \sum_{j, \ell=1} \int_{\mathbb{R}^{d}} \int_{\mathbb{R}^{d}} \nabla_{y} \psi_{\ell}(y) D_{x}^{2} a(|x-y|) \nabla_{y} \bar{\psi}_{\ell}(y)\left|\psi_{j}(x)\right|^{2} d x d y \\
4 \sum_{j, \ell=1} \int_{\mathbb{R}^{d}} \int_{\mathbb{R}^{d}} \nabla_{x} \psi_{j}(x) D_{x}^{2} \phi(|x-y|) \nabla_{x} \bar{\psi}_{j}(x)\left|\psi_{\ell}(y)\right|^{2} d x d y \\
-8 \sum_{j, \ell=1} \int_{\mathbb{R}^{d}} \int_{\mathbb{R}^{d}} \operatorname{Im}\left(\bar{\psi}_{j}(x) \nabla_{x} \psi_{j}(x)\right) D_{x}^{2} a(|x-y|) \operatorname{Im}\left(\bar{\psi}_{\ell}(y) \nabla_{y} \psi_{\ell}(y)\right) d x d y
\end{array}
$$

and lastly to

$$
=2 \sum_{j, \ell=1} \int_{\mathbb{R}^{d}} \int_{\mathbb{R}^{d}}\left(H_{j \ell}^{1} D_{x}^{2} a(|x-y|) \overline{H_{j \ell}^{1}}+H_{j \ell}^{2} D_{x}^{2} a(|x-y|) \overline{H_{j \ell}^{2}}\right) d x d y,
$$

where we set

$$
\begin{aligned}
& H_{j \ell}^{1}:=\psi_{j}(t, x) \nabla_{y} \overline{\psi_{\ell}(t, y)}+\nabla_{x} \psi_{j}(t, x) \overline{\psi_{\ell}(t, y)} \\
& H_{j \ell}^{2}:=\psi_{j}(t, x) \nabla_{y} \psi_{\ell}(t, y)-\nabla_{x} \psi_{j}(t, x) \psi_{\ell}(t, y) .
\end{aligned}
$$

Thus by (3.24), and since $a$ is a convex function one achieves $\mathcal{I I}_{2}(t) \geqslant 0$, for any $t \in \mathbb{R}$. We claim further that

$$
2 \sum_{j, \ell=1}^{N} \int_{\mathbb{R}^{d}} \int_{\mathbb{R}^{d}} \Delta_{x} a^{\star}(x, y) \nabla_{x} m_{\psi_{j}}(t, x) \cdot \nabla_{y} m_{\psi_{\ell}}(t, y) d x d y+\mathcal{I I}_{2}(t) \geqslant 0 .
$$


In fact by (see for instance to [36]), we obtain

$$
\begin{aligned}
& -2 \sum_{j, \ell=1}^{N} \int_{\mathbb{R}^{d}} \int_{\mathbb{R}^{d}} \Delta_{x}^{2} a^{\star}(x, y) m_{\psi_{j}}(x) m_{\psi_{\ell}}(y) d x d y \\
= & -2 \sum_{j, \ell=1}^{N} \int_{\mathbb{R}^{d}} \int_{\mathbb{R}^{d}} \Delta_{x} \Delta_{y} a(|x-y|) m_{\psi_{j}}(x) m_{\psi_{\ell}}(y) d x d y \\
= & 2 \sum_{j, \ell=1}^{N} \int_{\mathbb{R}^{d}} \int_{\mathbb{R}^{d}} \nabla_{x} m_{\psi_{j}}(x) D_{x}^{2} a(|x-y|) \cdot \nabla_{y} m_{\psi_{\ell}}(y) d x d y,
\end{aligned}
$$

then the l.h.s. of (3.25) becomes equal to

$$
\begin{array}{r}
2 \sum_{j, \ell=1}^{N} \int_{\mathbb{R}^{d}} \int_{\mathbb{R}^{d}} \nabla_{x} m_{\psi_{j}}(x) D_{x}^{2} a(|x-y|) \cdot \nabla_{y} m_{\psi_{\ell}}(y) d x d y \\
+4 \sum_{j, \ell=1} \int_{\mathbb{R}^{d}} \int_{\mathbb{R}^{d}}\left(H_{j \ell}^{1} D_{x}^{2} a(|x-y|) \overline{H_{j \ell}^{1}}+H_{j \ell}^{2} D_{x}^{2} a(|x-y|) \overline{H_{j \ell}^{2}}\right) d x d y .
\end{array}
$$

A straight computation displays

$$
\begin{array}{r}
2 \nabla_{x}\left|\psi_{j}(x)\right|^{2} D_{x}^{2} a(|x-y|) \cdot \nabla_{y}\left|\psi_{\ell}(y)\right|^{2} \\
+2 H_{j \ell}^{1} D_{x}^{2} a(|x-y|) \overline{H_{j \ell}^{1}}+2 H_{j \ell}^{2} D_{x}^{2} a(|x-y|) \overline{H_{j \ell}^{2}} \\
=2 H_{j \ell}^{1} D_{x}^{2} a(|x-y|) \overline{H_{j \ell}^{1}}-2 H_{j \ell}^{2} D_{x}^{2} a(|x-y|) \overline{H_{j \ell}^{2}} \\
+2 H_{j \ell}^{1} D_{x}^{2} a(|x-y|) \overline{H_{j \ell}^{1}}+2 H_{j \ell}^{2} D_{x}^{2} a(|x-y|) \overline{H_{j \ell}^{2}}=4 H_{j \ell}^{1} D_{x}^{2} a(|x-y|) \overline{H_{j \ell}^{1}} \geqslant 0 .
\end{array}
$$

where we employed along the calculation that the matrix $D_{x}^{2} a^{\star}=-D_{x y}^{2} a^{\star}=$ $D_{x}^{2} a^{\star}$ is symmetric. Gathering together (3.26) and (3.27) we have that (3.25) is satisfied. With this last inequality in mind, we sum now $\mathcal{I I}_{1}(t)$ with $\mathcal{I I}_{2}(t)$ realizing that

$$
\begin{array}{r}
\ddot{\mathcal{I}}(t)=2 \sum_{j, \ell=1}^{N} \int_{\mathbb{R}^{d}} \int_{\mathbb{R}^{d}} \Delta_{x} a^{\star}(x, y) \nabla_{x} m_{\psi_{j}}(t, x) \cdot \nabla_{y} m_{\psi_{\ell}}(t, y) d x d y \\
+\mathcal{I I}_{2}(t)+N_{\left(p, a^{\star}\right)}^{C}(t)+N_{\left(p, a^{\star}\right)}^{H F}(t)+R_{\left(p, a^{\star}\right)}^{C}(t) \\
\geqslant N_{\left(p, a^{\star}\right)}^{C}(t)+N_{\left(2, a^{\star}\right)}^{H F}(t)+R_{\left(p, a^{\star}\right)}^{C}(t),
\end{array}
$$

that is the desired (3.14).

The proof of Lemma 3.2 accomplishes also the following proposition.

Proposition 3.3. Let $\left(\psi_{j}\right)_{j=1}^{N} \in \mathcal{C}\left(\mathbb{R}, H^{1}\left(\mathbb{R}^{d}\right)^{N}\right)$ be as in Proposition 2.1 and $N_{\left(p, a^{\star}\right)}^{C}(t), N_{\left(2, a^{\star}\right)}^{H F}(t), R_{\left(p, a^{\star}\right)}^{C}(t)$ as in Lemma 3.2, then the following holds. 
- (Low regularity Morawetz interaction inequality)

$$
\begin{array}{r}
\ddot{\mathcal{I}}(t) \geqslant N_{\left(p, a^{\star}\right)}^{C}(t)+N_{\left(2, a^{\star}\right)}^{H F}(t)+R_{\left(p, a^{\star}\right)}^{C}(t) \\
+2 \sum_{j, \ell=1}^{N} \int_{\mathbb{R}^{d}} \int_{\mathbb{R}^{d}} \Delta_{x} a^{\star}(x, y) \nabla_{x} m_{\psi_{j}}(x) \cdot \nabla_{y} m_{\psi_{\ell}}(y) d x d y .
\end{array}
$$

- (High regularity Morawetz interaction inequality)

$$
\begin{array}{r}
\ddot{\mathcal{I}}(t) \geqslant N_{\left(p, a^{\star}\right)}^{C}(t)+N_{\left(2, a^{\star}\right)}^{H F}(t)+R_{\left(p, a^{\star}\right)}^{C}(t) \\
-2 \sum_{j, \ell=1}^{N} \int_{\mathbb{R}^{d}} \int_{\mathbb{R}^{d}} \Delta_{x}^{2} a^{\star}(x, y) m_{\psi_{j}}(x) m_{\psi_{\ell}}(y) d x d y .
\end{array}
$$

Proof. We will supply the proof in few lines. Here we shall make use of (3.28) along with (3.20), arriving to the inequalities (3.29) and (3.30).

A direct consequence of Lemma 3.2 is that we can prove the following:

Proposition 3.4. Assume $d \geqslant 1, p>2$ and let $\left(\psi_{j}\right)_{j=1}^{N} \in \mathcal{C}\left(\mathbb{R}, H^{1}\left(\mathbb{R}^{d}\right)^{N}\right)$ be as in Proposition 2.1. Then, selecting $a^{\star}(x, y)=|x-y|$, one has the global estimate

$$
\int_{\mathbb{R}} N_{\left(p, a^{\star}\right)}^{C}(t) d t \leqslant C \sum_{j=1}^{N}\left\|\psi_{j, 0}\right\|_{H_{x}^{1}}^{4},
$$

with $N_{\left(p, a^{\star}\right)}^{C}(t)$ as in (3.15). Moreover, let be $\mathcal{Q}_{\tilde{x}}^{d}(r)=\tilde{x}+[-r, r]^{d}$, with $r>0$ and $\tilde{x} \in \mathbb{R}^{d}$, one gets the following localized estimates: for $d \geqslant 2$,

$$
\begin{array}{r}
\left.\sum_{j, k, \ell=1}^{N} \widetilde{\lambda}_{j k} \int_{\mathbb{R}} \sup _{\tilde{x} \in \mathbb{R}^{d}} \int_{\left(\mathcal{Q}_{\tilde{x}}^{d}(r)\right)^{3}}\left|\psi_{j}(t, x)\right|^{p}|| \psi_{\ell}(t, y)\right|^{2}\left|\psi_{k}(t, z)\right|^{p} d x d y d z d t, \\
\leqslant C \sum_{j=1}^{N}\left\|\psi_{j, 0}\right\|_{H_{x}^{1}}^{4},
\end{array}
$$

where $\widetilde{\lambda}_{j k}=4 \lambda_{j k}(p-2) / p$ and $\left(\mathcal{Q}_{\tilde{x}}^{d}(r)\right)^{3}=\mathcal{Q}_{\tilde{x}}^{d}(r) \times \mathcal{Q}_{\tilde{x}}^{d}(r) \times \mathcal{Q}_{\tilde{x}}^{d}(r)$; for $d=1$,

$$
\begin{array}{r}
\sum_{j, k, \ell=1}^{N} \widetilde{\lambda}_{j k} \int_{\mathbb{R}} \sup _{\tilde{x} \in \mathbb{R}} \int_{\left(\mathcal{Q}_{\tilde{x}}^{1}(r)\right)^{2}}\left|\psi_{j}(t, x)\right|^{p}\left|\psi_{\ell}(t, x)\right|^{2}\left|\psi_{k}(t, z)\right|^{p} d x d z d t \\
\leqslant C \sum_{j=1}^{N}\left\|\psi_{j, 0}\right\|_{H_{x}^{1}}^{4},
\end{array}
$$

with $\left(\mathcal{Q}_{\tilde{x}}^{1}(r)\right)^{2}=\mathcal{Q}_{\tilde{x}}^{1}(r) \times \mathcal{Q}_{\tilde{x}}^{1}(r)$. 
Proof. We will use, there, the interaction inequality (3.14) with $N_{\left(2, a^{\star}\right)}^{H F}(t)=$ 0 because of $\beta=0$. Let us start by handling (3.16). Namely, by means of

$$
\nabla_{x} a^{\star}(x, y)=\frac{x-y}{|x-y|},
$$

and inspired by [29], we can write,

$$
\begin{aligned}
& \sum_{j, k, \ell=1}^{N} \lambda_{j k}^{*} \int_{\mathbb{R}^{d}} \int_{\mathbb{R}^{d}} \int_{\mathbb{R}^{d}} \frac{(x-y) \cdot(x-z)\left|\psi_{j}(x)\right|^{p}\left|\psi_{k}(z)\right|^{p}}{|x-y||x-z|^{d-\gamma_{1}+2}} m_{\psi_{\ell}}(y) d x d y d z, \\
& \quad=\frac{1}{2} \sum_{j, k=1}^{N} \lambda_{j k}^{*} \int_{\mathbb{R}^{d}} \int_{\mathbb{R}^{d}} \frac{1}{|x-z|^{d-\gamma_{1}+2}}\left|\psi_{j}(x)\right|^{p}\left|\psi_{k}(z)\right|^{p} K(x, z) d x d z,
\end{aligned}
$$

with $\lambda_{j k}^{*}=8 p \lambda_{j k}\left(d-\gamma_{1}\right) / d$ and where

$$
K(x, z)=(x-z) \cdot \sum_{\ell=1}^{N} \int_{\mathbb{R}^{d}} m_{\psi_{\ell}}(y)\left(\frac{x-y}{|x-y|}-\frac{z-y}{|z-y|}\right) d y .
$$

Then, the elementary inequality

$$
\begin{array}{r}
(x-z) \cdot\left(\frac{x-y}{|x-y|}-\frac{z-y}{|z-y|}\right) \\
=(|x-y||z-y|-(x-y) \cdot(z-y))\left(\frac{|x-y|+|z-y|}{|x-y||z-y|}\right) \geqslant 0,
\end{array}
$$

bears to

$$
\inf _{(x, y) \in \mathbb{R}^{d} \times \mathbb{R}^{d}} K(x, z) \geqslant 0 .
$$

By combining now the previous (3.37) with (3.35) we obtain that $R_{\left(p, a^{\star}\right)}^{C}(t) \geqslant$ 0 for any $t \in \mathbb{R}$. Then we achieved, at this stage, the following pointwise (in time) estimate

$$
N_{\left(p, a^{\star}\right)}^{C}(t) \leqslant \ddot{\mathcal{I}}(t)
$$

which, after an integration w.r.t. time variable over the interval $\left[t_{1}, t_{2}\right] \subseteq \mathbb{R}$ with $t_{1}, t_{2} \in \mathbb{R}$, becomes

$$
\int_{t_{1}}^{t_{2}} N_{(p,|x-y|)}^{C}(t) d t \lesssim \sup _{t \in\left[t_{1}, t_{2}\right]}|\dot{\mathcal{I}}(t)| .
$$

We have also the following

$$
\begin{aligned}
\sup _{t \in\left[t_{1}, t_{2}\right]}|\dot{\mathcal{I}}(t)| \leqslant 2 \sup _{t \in\left[t_{1}, t_{2}\right]} \sum_{j, \ell=1}^{N} \mid & \left|\int_{\mathbb{R}^{d}} \int_{\mathbb{R}^{d}} j_{\psi_{j}}(t, x) \cdot \nabla_{x} a^{\star}(x, y) m_{\psi_{\ell}}(t, y) d x d y\right| \\
& \lesssim \sup _{t \in\left[t_{1}, t_{2}\right]} \sum_{j=1}^{N}\left\|\psi_{j}(t)\right\|_{H_{x}^{1}}^{4} \lesssim \sum_{j=1}^{N}\left\|\psi_{j, 0}\right\|_{H_{x}^{1}}^{4}<\infty,
\end{aligned}
$$


for the reason that the $H_{x}^{1}$-norm of the solution is bounded according to the conservation laws (2.1), and (2.2). From the estimates (3.39), (3.38) and allowing $t_{1} \rightarrow-\infty, t_{2} \rightarrow+\infty$ we finally get (3.31) which displays, after recalling that

$$
\Delta_{x}|x-y|= \begin{cases}\frac{d-1}{|x-y|} & \text { if } \quad d \geqslant 2 \\ 2 \pi \delta_{x=y} & \text { if } \quad d=1\end{cases}
$$

as

$$
\begin{aligned}
\sum_{j, k, \ell=1}^{N} \widetilde{\lambda}_{j k} \int_{\mathbb{R}} \int_{\mathbb{R}^{3 d}} \frac{d-1}{|x-y||x-z|^{d-\gamma_{1}}}\left|\psi_{j}(x)\right|^{p}\left|\psi_{\ell}(y)\right|^{2}\left|\psi_{k}(z)\right|^{p} d x d y d z, \\
\\
\lesssim \sum_{j=1}^{N}\left\|\psi_{j, 0}\right\|_{H_{x}^{1}}^{4},
\end{aligned}
$$

with $\mathbb{R}^{3 d}=\mathbb{R}^{d} \times \mathbb{R}^{d} \times \mathbb{R}^{d}$, for $d \geqslant 2$ and

$$
\begin{array}{r}
\sum_{j, k, \ell=1}^{N} \widetilde{\lambda}_{j k} \int_{\mathbb{R}} \int_{\mathbb{R}^{2}} \frac{1}{|x-z|^{1-\gamma_{1}}}\left|\psi_{j}(x)\right|^{p}\left|\psi_{\ell}(x)\right|^{2}\left|\psi_{k}(z)\right|^{p} d x d z \\
\lesssim \sum_{j=1}^{N}\left\|\psi_{j, 0}\right\|_{H_{x}^{1}}^{4}
\end{array}
$$

for $d=1$. We are in position to go over the proof of (3.32) and (3.33). We notice that, for any $\tilde{x} \in \mathbb{R}^{d}$,

$$
\inf _{x, y, z \in \mathcal{Q}_{\tilde{x}}^{d}(r)}\left(\frac{1}{|x-y|}, \frac{1}{|z-y|}\right)=\inf _{x, y, z \in \mathcal{Q}_{0}^{d}(r)}\left(\frac{1}{|x-y|}, \frac{1}{|z-y|}\right)>0
$$

as an outcome, we can bound the l.h.s. of (3.40) as

$$
\sum_{j, k, \ell=1}^{N} \widetilde{\lambda}_{j k} \int_{\mathbb{R}^{d}} \int_{\mathbb{R}^{3 d}} \frac{d-1}{|x-y||x-z|^{d-\gamma_{1}}}\left|\psi_{j}(x)\right|^{p}\left|\psi_{\ell}(y)\right|^{2}\left|\psi_{k}(z)\right|^{p} d x d y d z d t
$$

$$
\left.\gtrsim \sum_{j, k, \ell=1}^{N} \widetilde{\lambda}_{j k} \int_{\mathbb{R}} \sup _{\tilde{x} \in \mathbb{R}^{d}} \int_{\left(\mathcal{Q}_{\tilde{x}}^{d}(r)\right)^{3}}\left|\psi_{j}(t, x)\right|^{p}|| \psi_{\ell}(t, y)\right|^{2}\left|\psi_{k}(t, z)\right|^{p} d x d y d z d t .
$$

Then the previous (3.40) and (3.43) guarantee that the estimate (3.32) holds. In a similar way we can manage the l.h.s of (3.41). To be specific we have, 
by utilizing again (3.42), that

$$
\begin{aligned}
& \sum_{j, k, \ell=1}^{N} \widetilde{\lambda}_{j k} \int_{\mathbb{R}} \int_{\mathbb{R}^{2}} \frac{d-1}{|x-y||x-z|^{1-\gamma_{1}}}\left|\psi_{j}(x)\right|^{p}\left|\psi_{\ell}(x)\right|^{2}\left|\psi_{k}(z)\right|^{p} d x d z d t \\
& \left.\gtrsim \sum_{j, k, \ell=1}^{N} \widetilde{\lambda}_{j k} \int_{\mathbb{R}} \sup _{\tilde{x} \in \mathbb{R}} \int_{\left(\mathcal{Q}_{\tilde{x}}^{1}(r)\right)^{2}}\left|\psi_{j}(t, x)\right|^{p}|| \psi_{\ell}(t, x)\right|^{2}\left|\psi_{k}(t, z)\right|^{p} d x d z d t,
\end{aligned}
$$

The above (3.41) and (3.44) give the way to (3.33). The proof of the proposition is finally completed.

In addition we get also the following result for the pure HF:

Proposition 3.5. Assume $d \geqslant 3, p=2$ and let $\left(\psi_{j}\right)_{i=1}^{N} \in \mathcal{C}\left(\mathbb{R}, H^{1}\left(\mathbb{R}^{n}\right)^{N}\right)$ be as in Proposition 2.1. Then we have, if one chooses $a^{\star}(x, y)=|x-y|$,

$$
-\sum_{j, \ell=1}^{N} \int_{\mathbb{R}} \int_{\mathbb{R}^{d}} \int_{\mathbb{R}^{d}} \Delta_{x}^{2} a^{\star}(x, y)\left|\psi_{j}(t, x)\right|^{2}\left|\psi_{\ell}(t, y)\right|^{2} d x d y d t \leqslant C \sum_{i=1}^{N}\left\|\psi_{i, 0}\right\|_{H_{x}^{1}}^{4} .
$$

Let be $\mathcal{Q}_{\tilde{x}}^{d}(r)=\tilde{x}+[-r, r]^{d}$, with $r>0$ and $\tilde{x} \in \mathbb{R}^{d}$, one gets the following estimates:

- for $d=3$

$$
\sum_{j=1}^{N} \int_{\mathbb{R}} \sup _{\tilde{x} \in \mathbb{R}^{3}} \int_{\mathcal{Q}_{\tilde{x}}^{3}(r)}\left|\psi_{j}(t, x)\right|^{4} d x d t \leqslant C \sum_{j=1}^{N}\left\|\psi_{j, 0}\right\|_{H_{x}^{1}}^{4}
$$

- for $d \geqslant 4$

$$
\begin{aligned}
& \sum_{j, \ell=1}^{N} \int_{\mathbb{R}} \sup _{\tilde{x} \in \mathbb{R}^{d}} \int_{\left(\mathcal{Q}_{\tilde{x}}^{d}(r)\right)^{2}}\left|\psi_{j}(t, x)\right|^{2}\left|\psi_{\ell}(t, y)\right|^{2} d x d y d t \leqslant C \sum_{j=1}^{N}\left\|\psi_{j, 0}\right\|_{H_{x}^{1}}^{4}, \\
& \text { with }\left(\mathcal{Q}_{\tilde{x}}^{d}(r)\right)^{2}=\mathcal{Q}_{\tilde{x}}^{d}(r) \times \mathcal{Q}_{\tilde{x}}^{d}(r) .
\end{aligned}
$$

Proof. We notice from the steps above that in the case $p=2$, the term $N_{(2,|x-y|)}^{C}(t)$ will vanish and $R_{(2,|x-y|)}^{C}(t) \geqslant 0$. We move now on the term (3.17) having the following

$$
2 \beta\left(d-\gamma_{2}\right) \int_{\mathbb{R}^{d}} \int_{\mathbb{R}^{d}} \frac{1}{|x-z|^{d-\gamma_{2}+2}}\left(\eta(x) \eta(z)-|\eta(x, z)|^{2}\right) K(x, z) d x d y,
$$

for $K(x, z)$ as in (3.36) and where we indicated by

$$
\eta(x, z)=\sum_{j=1}^{N} \psi_{j}(x) \bar{\psi}_{j}(z), \quad \eta(x)=\eta(x, x) .
$$

In addition we infer, by an use of the Cauchy-Schwartz inequality, the bound $|\eta(x, z)|^{2} \leqslant \eta(x) \eta(z)$, for any $x, y \in \mathbb{R}^{d}$. This observation, jointly again with 
(3.37), implies $N_{(2,|x-y|}^{H F}(t) \geqslant 0$ for any $t \in \mathbb{R}$. Hence, by applying the high regularity interaction inequality (3.30) and then arguing as in (3.38) and (3.39), one can easily attain the (3.45), which reads, by recalling that

$$
\Delta_{x}^{2}|x-y|=-\frac{(d-1)(d-3)}{|x-y|^{3}}, \quad \Delta_{x}^{2}|x-y|=-4 \pi \delta_{x=y} \leqslant 0,
$$

as

$$
\sum_{j, \ell=1}^{N} \int_{\mathbb{R}} \int_{\mathbb{R}^{d}} \int_{\mathbb{R}^{d}} \frac{\left|\psi_{j}(t, x)\right|^{2}\left|\psi_{\ell}(t, y)\right|^{2}}{|x-y|^{3}} d x d t \lesssim \sum_{j=1}^{N}\left\|\psi_{\mathrm{J}, 0}\right\|_{H_{x}^{1}}^{4},
$$

for $d \geqslant 4$ and

$$
\sum_{j, \ell=1}^{N} \int_{\mathbb{R}} \int_{\mathbb{R}^{3}}\left|\psi_{j}(t, x)\right|^{2}\left|\psi_{\ell}(t, x)\right|^{2} d x d t \lesssim \sum_{j=1}^{N}\left\|\psi_{j, 0}\right\|_{H_{x}^{1}}^{4},
$$

for $d=3$. The proofs of (3.46) and (3.47) are exactly the same as in Proposition 3.4, considering also the bound (3.42).

By the low and high regularity Morawetz interaction inequalities (3.29), (3.30), the Propositions 3.4 and 3.5 and taking into account that $N_{\left(p, a^{\star}\right)}^{C}(t) \geqslant$ 0 , one arrives at the following corollary, where some new linear correlationtype estimates associated to (1.1) are achieved. We have thus:

Corollary 3.6. Let $\left(\psi_{j}\right)_{j=1}^{N} \in \mathcal{C}\left(\mathbb{R}, H^{1}\left(\mathbb{R}^{n}\right)^{N}\right)$ be as in Proposition 2.1. Then one has, assuming $d \geqslant 1$ and $p>2$ such that (1.3) holds,

$$
\sum_{j=1}^{N}\left\|(-\Delta)^{\frac{1-d}{4}} \nabla\left|\psi_{j}(t, x)\right|^{2}\right\|_{L^{2}\left(\left(t_{1}, t_{2}\right) ; L_{x}^{2}\right)}^{2} \lesssim \sup _{t \in\left[t_{1}, t_{2}\right]}|\dot{\mathcal{I}}(t)| .
$$

In particular the following estimates are valid with $p \geqslant 2, \beta \geqslant 0$ and $\lambda_{j k} \geqslant 0$ :

- for $d=3$,

$$
\sum_{j=1}^{N}\left\|\psi_{j}(t, x)\right\|_{L^{4}\left(\left(t_{1}, t_{2}\right) ; L_{x}^{4}\right)}^{4} \lesssim \sup _{t \in\left[t_{1}, t_{2}\right]}|\dot{\mathcal{I}}(t)| ;
$$

- for $d \geqslant 4$,

$$
\sum_{j=1}^{N}\left\|(-\Delta)^{\frac{3-d}{4}}\left|\psi_{j}(t, x)\right|^{2}\right\|_{L^{2}\left(\left(t_{1}, t_{2}\right) ; L_{x}^{2}\right)}^{2} \lesssim \sup _{t \in\left[t_{1}, t_{2}\right]}|\dot{\mathcal{I}}(t)| .
$$

\section{The Decay of solutions to (1.1)}

Our main purpose in this section is to exhibit some decaying properties of the solution to (1.1) which is a essential property for the study of the scattering phenomena. With the aim of doing that, we present thus the proof of the of Theorem 1.1 and of the associated property (1.12) in Corollary 1.5. 
Proof of Theorem (1.1). Let us set $u(t, x)=\left(\psi_{j}(t, x)\right)_{j=1}^{N}$, utilizing both notations where it is needed. We split the proof in three different parts:

Case $p>2, d \geqslant 2$. It is sufficient to prove the property (1.8) for a suitable $2<r<2 d /(d-2)$ (for $2<r<+\infty$, if $d=2$ ), since the thesis for the general case can be acquired by the conservation of mass (2.1), the kinetic energy (2.2) and then by interpolation. Let us select $r=(2 d+8) /(d+2)$, we need to prove then

$$
\lim _{t \rightarrow \pm \infty}\|\psi(t)\|_{\mathcal{L}_{x}^{\frac{2 d+8}{d+2}}}=0 .
$$

We treat only the case $t \rightarrow \infty$, the case $t \rightarrow-\infty$ can be dealt analogously. Proceeding now by absurd as in [7] (we also to [40]), we assume that there exists a sequence $\left\{t_{n}\right\}$ with $t_{n} \rightarrow+\infty$ and a $\delta_{0}>0$

$$
\inf _{n}\left\|\psi\left(t_{n}, x\right)\right\|_{\mathcal{L}_{x}^{\frac{2 d+8}{d+2}}}=\delta_{0}
$$

Next we will make an use of the localized Gagliardo-Nirenberg inequality given in the Appendix A with $r=1$ and $\nu=2$ :

$$
\|\phi\|_{\mathcal{L}_{x}^{\frac{2 d+8}{d+2}}}^{\frac{2 d+8}{d+2}} \leqslant C\left(\sup _{\widetilde{x} \in \mathbb{R}^{d}}\|\phi\|_{\mathcal{L}^{2}\left(\mathcal{Q}_{\widetilde{x}}^{d}(1)\right)}\right)^{\frac{2}{d+4}}\|\phi\|_{\mathcal{H}_{x}^{1}}^{\frac{d+2}{d+4}},
$$

where $\mathcal{Q}_{\widetilde{x}}^{d}(1)$ is the unit cube in $\mathbb{R}^{d}$ centered in $\widetilde{x}$. By combining (4.2), (4.3), where we selected $\phi=u\left(t_{n}, x\right)$, with the bound $\left\|u\left(t_{n}, x\right)\right\|_{\mathcal{H}_{x}^{1}}<+\infty$, we notice that there exists $x_{n} \in \mathbb{R}^{d}$ and a $\varepsilon_{0}>0$ such that

$$
\inf _{n}\left\|u\left(t_{n}, x\right)\right\|_{\mathcal{L}^{2}\left(\mathcal{Q}_{x_{n}}^{d}(1)\right)}=\varepsilon_{0} .
$$

We can assert now that there exists $t^{*}>0$ such that

$$
\|u(t, x)\|_{\mathcal{L}^{2}\left(\mathcal{Q}_{x_{n}}^{d}(2)\right)} \geqslant \varepsilon_{0} / 2,
$$

for all $t \in\left(t_{n}, t_{n}+t^{*}\right)$ and where $\mathcal{Q}_{x_{n}}^{d}(2)$ denotes the cube in $\mathbb{R}^{d}$ with sidelenght 2 centered at $x_{n}$. Then $(4.5)$ can be showed as follows. Fix a cut-off function $\varphi(x) \in C_{0}^{\infty}\left(\mathbb{R}^{d}\right)$, so as $\varphi(x)=1$ for $x \in \mathcal{Q}_{0}^{d}(1)$ and $\varphi(x)=0$ for $x \notin \mathcal{Q}_{0}^{d}(2)$. Then by applying (3.2) where we choose $a(x)=\varphi\left(x-x_{n}\right)$ we get

$$
\left.\left|\frac{d}{d t} \int_{\mathbb{R}^{d}} \varphi\left(x-x_{n}\right)\right| u(t, x)\right|^{2} d x \mid \lesssim \sup _{t}\|u(t, x)\|_{\mathcal{H}_{x}^{1}}^{2} .
$$

Consequently, by (2.2) and the fundamental theorem of calculus we deduce

$$
\left.\left|\int_{\mathbb{R}^{d}} \varphi\left(x-x_{n}\right)\right| u(\sigma, x)\right|^{2} d x-\int_{\mathbb{R}^{d}} \varphi\left(x-x_{n}\right)|u(t, x)|^{2} d x|\leqslant \widetilde{C}| t-\sigma \mid,
$$

for a $\widetilde{C}>0$ which does not depend on $n$. Hence if we choose $t=t_{n}$ we get the elementary inequality

$$
\int_{\mathbb{R}^{d}} \varphi\left(x-x_{n}\right)|u(\sigma, x)|^{2} d x \geqslant \int_{\mathbb{R}^{d}} \varphi\left(x-x_{n}\right)\left|u\left(t_{n}, x\right)\right|^{2} d x-\widetilde{C}\left|t_{n}-\sigma\right|
$$


which implies, having in mind the support property of the function $\varphi$,

$$
\int_{\mathcal{Q}_{x_{n}}^{d}(2)}|u(\sigma, x)|^{2} d x \geqslant \int_{\mathcal{Q}_{x_{n}}^{d}(1)}\left|u\left(t_{n}, x\right)\right|^{2} d x-\widetilde{C}\left|t_{n}-\sigma\right| .
$$

Hence (4.5) follows by an application of (4.4), provided that we pick up $t^{*}>0$ such that $3 \varepsilon_{0}^{2}-4 \widetilde{C} t^{*}>0$. The inequality (4.5) is in contradiction with the Morawetz estimates (3.32). In fact, the lower bound (4.5) means that

$$
\inf _{n}\left(\inf _{t \in\left(t_{n}, t_{n}+t^{*}\right)} \sum_{j=1}^{N}\left\|\psi_{j}(t)\right\|_{L_{x}^{2}\left(\mathcal{Q}_{x_{n}}^{d}(2)\right)}^{2}\right) \gtrsim \varepsilon_{0}^{2}>0
$$

with $t^{*}$ as above and the time intervals $\left(t_{n}, t_{n}+t^{*}\right)$ chosen to be disjoint. By Hölder inequality we attain also

$$
\inf _{n}\left(\inf _{t \in\left(t_{n}, t_{n}+t^{*}\right)} \sum_{j=1}^{N}\left\|\psi_{j}(t)\right\|_{L_{x}^{p}\left(\mathcal{Q}_{x_{n}}^{d}(2)\right)}^{p}\right) \gtrsim \varepsilon_{0}^{2}>0 .
$$

Thus we can formulate the following

$$
\begin{array}{r}
\sum_{j, k, \ell=1}^{N} \widetilde{\lambda}_{j k} \int_{\mathbb{R}} \sup _{\tilde{x} \in \mathbb{R}^{d}} \int_{\left(\mathcal{Q}_{\tilde{x}}^{d}(2)\right)^{3}}\left|\psi_{j}(t, x)\right|^{p}\left|\psi_{\ell}(t, y)\right|^{2}\left|\psi_{k}(t, z)\right|^{p} d x d y d z d t \\
\gtrsim \sum_{j, k, \ell=1}^{N} \widetilde{\lambda}_{j k} \int_{\mathbb{R}} \int_{\left(\mathcal{Q}_{x_{n}}^{d}(2)\right)^{3}}\left|\psi_{j}(t, x)\right|^{p}\left|\psi_{\ell}(t, y)\right|^{2}\left|\psi_{k}(t, z)\right|^{p} d x d y d z d t \\
\gtrsim \sum_{j, k=1}^{N} \widetilde{\lambda}_{j k} \sum_{n} \int_{t_{n}}^{t_{n}+t^{*}} \varepsilon_{0}^{6} d t \gtrsim \sum_{n} t^{*} \varepsilon_{0}^{6} d t=\infty,
\end{array}
$$

where in the last inequality we employed (4.5) in combination with (4.9) and (4.10). This brings us to contradiction with (3.32).

Case $p>2, d=1$. It can be handled in a similar manner, now by seeking for a $2<r<\infty$. By an application of the Hölder inequality, one figures out the bound

$$
\begin{aligned}
\sum_{j, k, \ell=1}^{N} \widetilde{\lambda}_{j k} \int_{\mathbb{R}} \sup _{\tilde{x} \in \mathbb{R}^{1}} \int_{\left(\mathcal{Q}_{\tilde{x}}^{1}(2)\right)^{2}}\left|\psi_{j}(t, x)\right|^{p}\left|\psi_{\ell}(t, x)\right|^{2}\left|\psi_{k}(t, z)\right|^{p} d x d z d t \\
\gtrsim \sum_{j, k=1}^{N} \widetilde{\lambda}_{j k} \sum_{n} \int_{t_{n}}^{t_{n}+t^{*}} \int_{\mathcal{Q}_{x_{n}}^{1}(2)} \int_{\mathcal{Q}_{x_{n}}^{1}(2)}\left|\psi_{j}(t, x)\right|^{p+2}\left|\psi_{k}(t, z)\right|^{p} d x d z d t \\
\gtrsim \sum_{j, k=1}^{N} \widetilde{\lambda}_{j k} \sum_{n} \int_{t_{n}}^{t_{n}+t^{*}} \varepsilon_{0}^{4} d t \gtrsim \sum_{n} t^{*} \varepsilon_{0}^{4} d t=\infty .
\end{aligned}
$$


Therefore, we can proceed as above, getting a contradiction with (3.33) instead. Lastly, the conservation law (1.6), (1.8) and the Gagliardo-Nirenberg inequality

$$
\left\|\psi_{j}(t)\right\|_{L_{x}^{\infty}}^{4} \lesssim\left\|\psi_{j}(t)\right\|_{L_{x}^{6}}^{3}\left\|\partial_{x} \psi_{j}(t)\right\|_{L_{x}^{2}}
$$

ensure

$$
\lim _{t \rightarrow+\infty}\left\|\psi_{j}(t)\right\|_{L_{x}^{\infty}}=0
$$

for any $j=1, \ldots, N$.

Case $p=2, d \geqslant 3$. We follow the same lines of the proof above. However, one can not use, at this level, the Proposition 3.4 because we are picking up $p=2$. Then we are forced to focus on the Proposition 3.5: for $d \geqslant 4$, we make use of (4.9) attaining

$$
\begin{array}{r}
\sum_{j, \ell=1}^{N} \int_{\mathbb{R}} \sup _{\tilde{x} \in \mathbb{R}^{d}} \int_{\mathcal{Q}_{\tilde{x}}^{d}(2)} \int_{\mathcal{Q}_{\tilde{x}}^{d}(2)}\left|\psi_{j}(t, x)\right|^{2}\left|\psi_{\ell}(t, y)\right|^{2} d x d y d t \\
\gtrsim \sum_{j, \ell=1}^{N} \sum_{n} \int_{t_{n}}^{t_{n}+t^{*}} \int_{\mathcal{Q}_{x_{n}}^{d}(2)} \int_{\mathcal{Q}_{x_{n}}^{d}(2)}\left|\psi_{j}(t, x)\right|^{2}\left|\psi_{\ell}(t, y)\right|^{2} d x d y d t \\
\gtrsim \sum_{n} \int_{t_{n}}^{t_{n}+t^{*}} \varepsilon_{0}^{4} d t=\sum_{n} t^{*} \varepsilon_{0}^{4} d t=\infty,
\end{array}
$$

which contradicts (3.47). In the same manner we can treat the case $d=3$. In fact by Hölder inequality and (4.9), we arrive at

$$
\begin{aligned}
\sum_{j=1}^{N} \int_{\mathbb{R}} \sup _{\tilde{x} \in \mathbb{R}^{3}} \int_{\mathcal{Q}_{\tilde{x}}^{3}(2)}\left|\psi_{j}(t, x)\right|^{4} d x d t & \gtrsim \sum_{j=1}^{N} \sum_{n} \int_{t_{n}}^{t_{n}+t^{*}} \int_{\mathcal{Q}_{x_{n}}^{3}(2)}\left|\psi_{j}(t, x)\right|^{2} d x d t \\
& \gtrsim \sum_{n} \int_{t_{n}}^{t_{n}+t^{*}} \varepsilon_{0}^{2} d t=\sum_{n} t^{*} \varepsilon_{0}^{2} d t=\infty
\end{aligned}
$$

that is in contradiction with (3.47). Then the proof is now complete.

\section{SCATtering FOR NLC AND NLHF Systems}

We carry out, along this section, the proof of Theorem 1.2 and the corresponding scattering property (1.13) in Corollary 1.5. Albeit these results are classic (we suggest [9], [20] and references therein for additional reading), here we disclose them in a more general and self-contained form. We recall from [25], also:

Definition 5.1. An exponent pair $(q, r)$ is Schrödinger-admissible if $2 \leqslant$ $q, r \leqslant \infty,(q, r, d) \neq(2, \infty, 2)$, and

$$
\frac{2}{q}+\frac{d}{r}=\frac{d}{2}
$$


Proposition 5.2. Let be two Schrödinger-admissible pairs $(q, r)$ and $(\widetilde{q}, \widetilde{r})$. Then we have for $\kappa=0,1$ and the following estimates:

$$
\begin{aligned}
\left\|\nabla^{\kappa} e^{-i t \Delta_{x}} g\right\|_{L_{t}^{q} L_{x}^{r}} & \left\|\nabla^{\kappa} \int_{0}^{t} e^{-i(t-\tau) \Delta_{x}} G(\tau) d \tau\right\|_{L_{t}^{q} L_{x}^{r}} \\
& \leqslant C\left(\left\|\nabla^{\kappa} g\right\|_{L_{x}^{2}}+\left\|\nabla^{\kappa} G\right\|_{L_{t}^{\widetilde{q}^{\prime}} L_{x}^{\widetilde{r}^{\prime}}}\right) .
\end{aligned}
$$

We want to prove Theorem 1.2, then we demand to gain the necessary space-time summability for the scattering. This is contained in the following:

Lemma 5.3. Assume $\left(\psi_{j}\right)_{j=1}^{N} \in \mathcal{C}\left(\mathbb{R}, \mathcal{H}_{x}^{1}\right)$ as in Theorem 1.2. Then we have

$$
\left(\psi_{j}\right)_{j=1}^{N} \in L^{q}\left(\mathbb{R}, \mathcal{W}_{x}^{1, r}\right)
$$

for every Schrödinger-admissible pair $(q, r)$.

Proof. We consider the integral operator associated to (1.1), that is

$$
u(t)=e^{i t \Delta_{x}} u_{0}+\int_{0}^{t} e^{i(t-\tau) \Delta_{x}} \Gamma(u(\tau), p) d \tau
$$

where $t>0$ and

$$
\begin{gathered}
u(t)=\left(\begin{array}{c}
\psi_{1}(t) \\
\vdots \\
\psi_{N}(t)
\end{array}\right), \quad u_{0}=\left(\begin{array}{c}
\psi_{1,0} \\
\vdots \\
\psi_{N, 0}
\end{array}\right) \\
\mathcal{G}(u, p)=\left(\begin{array}{c}
G\left(\psi_{1}, \psi_{k}\right) \\
\vdots \\
G\left(\psi_{N}, \psi_{k}\right)
\end{array}\right)
\end{gathered}
$$

We start by dealing with $p>2$ and choose $\left(q_{1}^{\prime}, r_{1}^{\prime}\right)$ so that

$$
\left(q_{1}, r_{1}\right):=\left(\frac{4 p}{d p-d-\gamma_{1}}, \frac{2 d p}{d+\gamma_{1}}\right)
$$

In this way the Strichartz estimates (5.2), the fractional chain rule, the Hölder and Hardy-Littlewood-Sobolev inequalities enhance, for $\kappa=0,1$, to the following (see [29]) 


$$
\begin{aligned}
& \lesssim \sum_{\kappa=1}^{2}\left\|\nabla^{\kappa} \psi_{j, 0}\right\|_{L_{x}^{2}}+\sum_{\kappa=1}^{2}\left\|\sum_{k=1}^{N} \lambda_{j k} \nabla^{\kappa}\left(\left[|x|^{-\left(d-\gamma_{1}\right)} *\left|\psi_{k}\right|^{p}\right]\left|\psi_{j}\right|^{p-2} \psi_{j}\right)\right\|_{L_{t>T}^{q_{1}^{\prime}} L_{x}^{r_{1}^{\prime}}}
\end{aligned}
$$

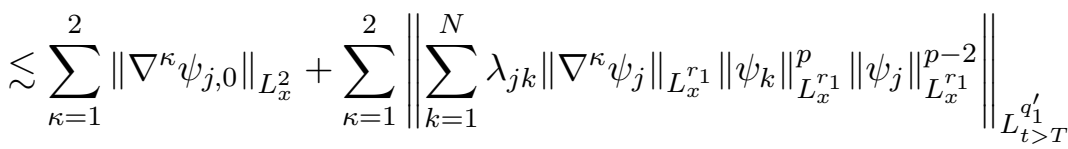

$$
\begin{aligned}
& +\left\|\sum_{k=1}^{N} \lambda_{j k}\left[|x|^{-\left(d-\gamma_{1}\right)} * \nabla\left|\psi_{k}\right|^{p}\right]\left|\psi_{j}\right|^{p-2} \psi_{j}\right\|_{L_{t>T}^{q_{1}^{\prime}} L_{x}^{r_{1}^{\prime}}}
\end{aligned}
$$

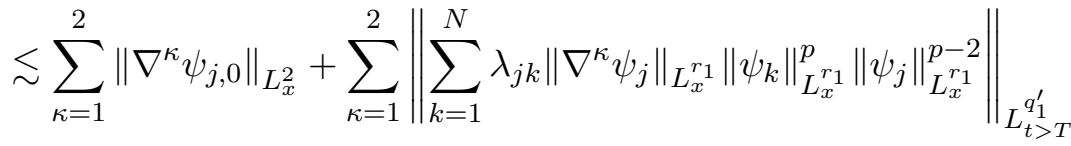

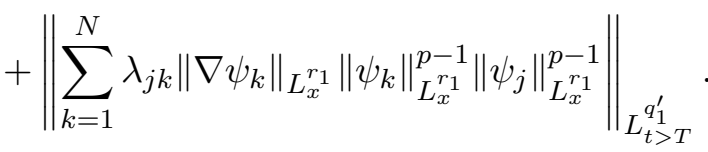

Summing up over $j=1 \ldots N$, we see that the last term of the previous inequality is not greater than

$$
\begin{array}{r}
\sum_{j=1}^{N} \sum_{\kappa=1}^{2}\left\|\nabla^{\kappa} \psi_{j, 0}\right\|_{L_{x}^{2}}+\sum_{j, k=1}^{N} \sum_{\kappa=1}^{2}\left\|\lambda_{j k}\right\| \nabla^{\kappa} \psi_{j}\left\|_{L_{x}^{r}}\right\| \psi_{k}\left\|_{L_{x}^{r_{1}}}^{p}\right\| \psi_{j}\left\|_{L_{x}^{r_{1}}}^{p-2}\right\|_{L_{t>T}^{q_{1}^{\prime}}} \\
+\sum_{j, k=1}^{N}\left\|\lambda_{j k}\right\| \nabla \psi_{k}\left\|_{L_{x}^{r_{1}}}\right\| \psi_{k}\left\|_{L_{x}^{r_{1}}}^{p-1}\right\| \psi_{j}\left\|_{L_{x}^{r_{1}}}^{p-1}\right\|_{L_{t>T}^{q_{1}^{\prime}} L_{x}^{r_{1}^{\prime}}} . \\
\lesssim\left\|u_{0}\right\|_{\mathcal{H}_{x}^{1}}+\sum_{\kappa=1}^{2}\|\| \nabla^{\kappa} u\left\|_{\mathcal{L}_{x}^{r_{1}}}\right\| u\left\|_{\mathcal{L}_{x}^{r_{1}}}^{(2 p-2)}\right\|_{L_{t>T}^{q_{1}^{\prime}}}
\end{array}
$$

We single out now $\theta_{1}=\left(q_{1}-q_{1}^{\prime}\right) /\left(2 p q_{1}^{\prime}-2 q_{1}^{\prime}\right) \in(0,1)$, because of (1.3) and (1.4). Furthermore, direct calculations show that

$$
\frac{1}{q_{1}^{\prime}}=\frac{2(p-1) \theta_{1}+1}{q_{1}}
$$


yielding for the last term on the r.h.s. of (5.7),

$$
\begin{array}{r}
\left\|u_{0}\right\|_{\mathcal{H}_{x}^{1}}+\sum_{\kappa=1}^{2}\|\| \nabla^{\kappa} u\left\|_{\mathcal{L}_{x}^{r_{1}}}\right\| u\left\|_{\mathcal{L}_{x}^{r_{1}}}^{(2 p-2)}\right\|_{L_{t>T}^{q_{1}^{\prime}}} \\
\lesssim\left\|u_{0}\right\|_{\mathcal{H}_{x}^{1}}+\|\| \nabla^{\kappa} u\left\|_{\mathcal{L}_{x}^{r_{1}}}\right\| u\left\|_{\mathcal{L}_{x}^{r_{1}^{1}}}^{(2 p-2)\left(1-\theta_{1}\right)}\right\| u\left\|_{\mathcal{L}_{x}^{r_{1}}}^{(2 p-2) \theta_{1}}\right\|_{L_{t>T}^{q_{1}^{\prime}}} \\
\lesssim\left\|u_{0}\right\|_{\mathcal{H}_{x}^{1}}+\|\| u\left\|_{\mathcal{W}_{x}^{1, r_{1}}}\right\| u\left\|_{\mathcal{L}_{x}^{r_{1}}}^{(2 p-2)\left(1-\theta_{1}\right)}\right\| u\left\|_{\mathcal{L}_{x}^{r_{1}}}^{(2 p-2) \theta_{1}}\right\|_{L_{t>T}^{q_{1}^{\prime}}} \\
\lesssim\left\|u_{0}\right\|_{\mathcal{H}_{x}^{1}}+\|\| u\left\|_{\mathcal{L}_{x}^{r 1}}^{(2 p-2)\left(1-\theta_{1}\right)}\right\| u\left\|_{\mathcal{W}_{x}^{1, r_{1}}}^{(2 p-2) \theta_{1}+1}\right\|_{L_{t>T}^{q_{1}^{\prime}}} \\
\leqslant C\left(\left\|u_{0}\right\|_{\mathcal{H}_{x}^{1}}+\|u\|_{L_{t>T}^{\infty} \mathcal{L}_{x}^{r_{1}}}^{(2 p-2)\left(1-\theta_{1}\right)}\|u\|_{L_{t>T}^{q_{1}} \mathcal{W}_{x}^{1, r_{1}}}^{(2 p-2) \theta_{1}+1}\right),
\end{array}
$$

with the constant $C>0$ independent from $t$ and $T$. An use of (5.6), (5.7), (5.8) leads to

$$
\|u\|_{L_{t>T}^{q_{1}} \mathcal{W}_{x}^{1, r_{1}}} \lesssim\left\|u_{0}\right\|_{\mathcal{H}_{x}^{1}}+\|u\|_{L_{t>T}^{\infty} \mathcal{L}_{x}^{r_{1}}}^{(2 p-2)\left(1-\theta_{1}\right)}\|u\|_{L_{t>T}^{q_{1}} \mathcal{W}_{x}^{1, r_{1}}}^{(2 p-2) \theta_{1}+1}
$$

where

$$
\lim _{T \rightarrow+\infty}\|u\|_{L_{t>T}^{\infty} \mathcal{L}_{x}^{r_{1}}=0}
$$

by (1.8) in Theorem 1.1. Then, picking up $T$ sufficiently large we infer that

$$
\|u\|_{L^{q_{1}}\left((T, t), \mathcal{W}_{x}^{1, r_{1}}\right)}<\infty
$$

and consequently that $u \in L^{q_{1}}\left((T,+\infty), \mathcal{W}_{x}^{1, r_{1}}\right)$. Likewise, we can earn $u \in L^{q_{1}}\left((-\infty,-T), \mathcal{W}_{x}^{1, r_{1}}\right)$. In conclusion, by a continuity argument and Strichartz estimates (5.1), one has $u \in L^{q}\left(\mathbb{R}, \mathcal{W}_{x}^{1, r}\right)$ for any Schrödingeradmissible pair $(q, r)$.

Let us manage $p=2$. We pick $\left(q_{2}^{\prime}, r_{2}^{\prime}\right)$ defined by

$$
\left(q_{2}, r_{2}\right):=\left(\frac{8}{d-\gamma_{1}}, \frac{4 d}{d+\gamma_{1}}\right)
$$


then we get, analogously as above,

$$
\begin{array}{r}
\sum_{j=1}^{N} \sum_{\kappa=1}^{2}\left\|\nabla^{\kappa} \psi_{j}\right\|_{L_{t}^{q_{2}} L_{x}^{r_{2}}} \lesssim \sum_{j=1}^{N} \sum_{\kappa=1}^{2}\left\|\nabla^{\kappa} \psi_{j, 0}\right\|_{L_{x}^{2}} \\
+\sum_{j, k=1}^{N} \sum_{\kappa=1}^{2}\left\|\nabla^{\kappa}\left(\lambda_{j k}\left[|x|^{-\left(d-\gamma_{1}\right)} *\left|\psi_{k}\right|^{2}\right] \psi_{j}\right)\right\|_{L_{t>T}^{q_{2}^{\prime}} L_{x}^{r_{2}^{\prime}}} \\
-\beta \sum_{j, k=1}^{N} \sum_{\kappa=1}^{2}\left\|\nabla^{\kappa}\left(\left[|x|^{-\left(d-\gamma_{2}\right)} *\left|\psi_{k}\right|^{2}\right] \psi_{j}-\left[|x|^{-\left(d-\gamma_{2}\right)} * \bar{\psi}_{k} \psi_{j}\right] \psi_{k}\right)\right\|_{L_{t>T}^{q_{2}^{\prime}} L_{x}^{r_{2}^{\prime}}} \\
\lesssim\left\|u_{0}\right\|_{\mathcal{H}_{x}^{1}}+\sum_{\kappa=1}^{2}\|\| \nabla^{\kappa} u\left\|_{\mathcal{L}_{x}^{r_{2}}}\right\| u\left\|_{\mathcal{L}_{x}^{r_{2}}}^{2}\right\|_{L_{t>T}^{q_{2}^{\prime}}} \\
\lesssim\left\|u_{0}\right\|_{\mathcal{H}_{x}^{1}}+\|\| u\left\|_{\mathcal{L}_{x}^{r_{2}}}^{2\left(1-\theta_{2}\right)}\right\| u\left\|_{\mathcal{W}_{x}^{1, r_{2}}}^{2 \theta_{2}+1}\right\|_{L_{t>T}^{q_{2}^{\prime}}} \\
\lesssim\left\|u_{0}\right\|_{\mathcal{H}_{x}^{1}}+\|u\|_{L_{t>T}^{\infty}}^{2\left(1-\theta_{2}\right)} \mathcal{L}_{x}^{r_{2}}\|u\|_{L_{t>T}^{q_{2}} \mathcal{W}_{x}^{1, r_{2}}}^{2 \theta_{2}},
\end{array}
$$

where $\theta_{2}=\left(q_{2}-q_{2}^{\prime}\right) / 2 q_{2}^{\prime} \in(0,1)$ and such that

$$
\frac{1}{q_{2}^{\prime}}=\frac{2 \theta_{1}+1}{q_{2}}
$$

This enables us to rewrite (5.10) as

$$
\|u\|_{L_{t>T}^{q_{2}} \mathcal{W}_{x}^{1, r_{2}}} \lesssim\left\|u_{0}\right\|_{\mathcal{H}_{x}^{1}}+\|u\|_{L_{t>T}^{\infty} \mathcal{L}_{x}^{r_{2}}}^{2\left(1-\theta_{2}\right)}\|u\|_{L_{t>T}^{q_{2}} \mathcal{W}_{x}^{1, r_{2}}}^{2 \theta_{2}+1}
$$

with

$$
\lim _{T \rightarrow+\infty}\|u\|_{L_{t>T}^{\infty} \mathcal{L}_{x}^{r_{2}}=0}
$$

again by (1.8) in Theorem 1.1. Thus one argues as in the previous lines carrying out again that $u \in L^{q}\left(\mathbb{R}, \mathcal{W}_{x}^{1, r}\right)$ for any admissible pair $(q, r)$.

Proof of Theorem 1.2. We exploit the proof of Theorem 1.2 for $p>2$ and $p=2$ in a unified manner. We start from:

Asymptotic completeness: We write $\widetilde{u}(t)=e^{-i t \Delta_{x}} u(t)$ getting then from (5.4)

$$
\widetilde{u}\left(t_{2}\right)-\widetilde{u}\left(t_{1}\right)=i \int_{t_{1}}^{t_{2}} e^{-i s \Delta_{x}} \mathcal{G}(u, p) d s
$$


An use of the Strichartz estimates (5.1) bears to

$$
\left\|\int_{t_{1}}^{t_{2}} e^{-i s \Delta_{x}} \mathcal{G}(u, p) d s\right\|_{\mathcal{H}_{x}^{1}}
$$

$$
\begin{array}{r}
\lesssim \sum_{j, k=1}^{N} \lambda_{j k}\left\|\left[|x|^{-\left(d-\gamma_{1}\right)} *\left|\psi_{k}\right|^{p}\right]\left|\psi_{j}\right|^{p-2} \psi_{j}\right\|_{L^{q_{1}^{\prime}\left(\left(t_{1}, t_{2}\right), W_{x}^{r_{1}^{\prime}}\right)}} \\
+\beta \sum_{j, k=1}^{N}\left\|\left[|x|^{-\left(d-\gamma_{2}\right)} *\left|\psi_{k}\right|^{2}\right] \psi_{j}-\left[|x|^{-\left(d-\gamma_{2}\right)} * \bar{\psi}_{k} \psi_{j}\right] \psi_{k}\right\|_{L^{q_{2}^{\prime}\left(\left(t_{1}, t_{2}\right), W_{x}^{r_{2}^{\prime}}\right)}},
\end{array}
$$

with $\left(q_{1}, r_{1}\right)$ and $\left(q_{2}, r_{2}\right)$ admissible pairs as in (5.5) and (5.9). Then it suffices to display that

$$
\lim _{t_{1}, t_{2} \rightarrow \infty}\left\|\widetilde{u}\left(t_{2}\right)-\widetilde{u}\left(t_{1}\right)\right\|_{\mathcal{H}_{x}^{1}}=0
$$

which is verified by (5.11) on condition that

$$
\begin{aligned}
& \lim _{t_{1}, t_{2} \rightarrow \infty} \sum_{j, k=1}^{N} \lambda_{j k}\left\|\left[|x|^{-\left(d-\gamma_{1}\right)} *\left|\psi_{k}\right|^{p}\right]\left|\psi_{j}\right|^{p-2} \psi_{j}\right\|_{L^{q_{1}^{\prime}\left(\left(t_{1}, t_{2}\right), W_{x}^{r_{1}^{\prime}}\right)}}=0 \\
& \lim _{t_{1}, t_{2} \rightarrow \infty} \sum_{j, k=1}^{N}\left\|\left[\left(\lambda_{j k}|x|^{-\left(d-\gamma_{1}\right)}+\beta|x|^{-\left(d-\gamma_{2}\right)}\right) *\left|\psi_{k}\right|^{2}\right] \psi_{j}\right\|_{L_{2}^{q_{2}^{\prime}\left(\left(t_{1}, t_{2}\right), W_{x}^{r_{2}^{\prime}}\right)}}=0 \\
& \beta \lim _{t_{1}, t_{2} \rightarrow \infty} \sum_{j, k=1}^{N}\left\|\left[|x|^{-\left(d-\gamma_{2}\right)} * \bar{\psi}_{k} \psi_{j}\right] \psi_{k}\right\|_{L^{q_{2}^{\prime}\left(\left(t_{1}, t_{2}\right), W_{x}^{r_{2}^{\prime}}\right)}}=0,
\end{aligned}
$$

which can be easily performed following the same lines of the proof of Lemma 5.3. One can see, as a final step, that there are $\left(\psi_{1,0}^{ \pm}, \ldots, \psi_{N, 0}^{ \pm}\right) \in H^{1}\left(\mathbb{R}^{d}\right)^{N}$ and a map $\left(\psi_{1}(t), \ldots, \psi_{N}(t)\right) \rightarrow\left(\psi_{1,0}^{ \pm}, \ldots, \psi_{N, 0}^{ \pm}\right)$in $H^{1}\left(\mathbb{R}^{d}\right)^{N}$ when $t \rightarrow \pm \infty$. Notice that, by Proposition 2.1, we establish also the following conservation laws

$$
\begin{array}{r}
M\left(\psi_{1,0}^{ \pm}, \ldots, \psi_{N, 0}^{ \pm}\right)=\left\|\left(\psi_{1,0}, \ldots, \psi_{N, 0}\right)\right\|_{\mathcal{L}_{x}^{2}}^{2} \\
\sum_{j=1}^{N} \int_{\mathbb{R}^{d}}\left(\left|\Delta \psi_{j, 0}^{ \pm}\right|^{2}+\kappa\left|\nabla \psi_{j, 0}^{ \pm}\right|\right) d x=E\left(\psi_{1,0}, \ldots, \psi_{N, 0}\right)
\end{array}
$$

Existence of wave operators: The construction of the wave operators comes from standard arguments, we refer to [9] for more details about the matter. Then we skip the proof.

Remark 5.4. Once (1.8) is achieved in the range $2<r<2 d /(d-2)$, we were able to set up the scattering operator in $H^{1}\left(\mathbb{R}^{d}\right)^{N}$, as we did in the previous section. Now, similarly [40], we arrive by Sobolev embedding at

$$
\left\|\psi_{j}(t)\right\|_{L_{x}^{\frac{2 d}{d-2}}} \lesssim\left\|\psi_{j}(t)-e^{i t \Delta} \psi_{j, 0}^{ \pm}\right\|_{H_{x}^{1}}+\left\|e^{i t \Delta} \psi_{j, 0}^{ \pm}\right\|_{L_{x}^{\frac{2 d}{d-2}}} .
$$


Now the above estimate (5.12) combined with the classical dispersive estimate for the free propagator

$$
\left\|e^{i t \Delta} \psi_{j, 0}^{ \pm}\right\|_{L_{x}^{\frac{2 d}{d-2}}} \lesssim \frac{1}{t}\left\|\psi_{j, 0}^{ \pm}\right\|_{L_{x}^{\frac{2 d}{d+2}}}
$$

again the Sobolev-embedding and (1.9), allow also to

$$
\lim _{t \rightarrow \infty}\left\|\psi_{j}(t)\right\|_{L_{x}^{\frac{2 d}{d-2}}}=0 .
$$

The proof of Theorem 1.1 is now completed.

\section{Appendix A. A Gagliardo-Nirenberg inequality}

The principal target of this section is to exhibit (4.3) that is a localized version of the Gagliardo-Nirenberg inequality which appears in the proof of Proposition 1.1. Although it is known so far in the literature in different forms (let us cite here [7], [40], [28] or [38] in the context of product space $\mathbb{R}^{d} \times M$, with $M$ a compact manifold), we show here a more general new one. We have:

Proposition A.1. Let be $d \geqslant 1, \mu \in \mathbb{N}$ and $\nu \in \mathbb{N} \cup\{0\}$, then for all vector-valued functions $\phi=\left(\phi_{\ell}\right)_{\ell=1}^{\mu} \in H^{1}\left(\mathbb{R}^{d}\right)^{\mu}$ one gets the following

$$
\|\phi\|_{L^{\frac{2 d+2 \nu+4}{d+\nu}}\left(\mathbb{R}^{d}\right)^{\mu}}^{\frac{2 d+2 \nu+4}{d+\nu}} \leqslant C\left(\sup _{x \in \mathbb{R}^{d}}\|\phi\|_{L^{2}\left(Q_{\tilde{x}}^{d}(r)\right)^{\mu}}\right)^{\frac{4}{d+\nu}}\|\phi\|_{H^{1}\left(\mathbb{R}^{d}\right)^{\mu}}^{2},
$$

with $\mathcal{Q}_{\widetilde{x}}^{d}(r)=\widetilde{x}+[-r, r]^{d}$ being a $r$ dilation of the unit cube centered at $\widetilde{x}$.

Proof. Fix $r>0$ and consider $\widetilde{x}_{s} \in \mathbb{R}^{d}$ connected to a covering of $\mathbb{R}^{d}$ given by a family of cubes $\left\{\mathcal{Q}_{\widetilde{x}_{s}}^{d}(r)\right\}_{s \in \mathbb{N}}$ such that meas $d\left(\mathcal{Q}_{\widetilde{x}_{s_{1}}}^{d}(r) \cup \mathcal{Q}_{\widetilde{x}_{s_{2}}}^{d}(r)\right)=0$ for $s_{1} \neq s_{2}$, where meas $_{d}$ is the Lebesgue measure in $\mathbb{R}^{d}$. Without loss of generality, we can take $\phi=\left(\phi_{\ell}\right)_{\ell=1}^{\mu}$, such that $\operatorname{supp}\left(\phi_{\ell}\right) \subseteq \mathcal{Q}_{\widetilde{x}_{s}}^{d}(r)$ for any $\ell=1, \ldots, \mu$, then by the classical Gagliardo-Nirenberg inequality we attain

$$
\sum_{\ell=1}^{\mu} \int_{\mathcal{Q}_{\widetilde{x}_{s}}^{d}(r)}\left|\phi_{\ell}\right|^{\frac{2 d+2 \nu+4}{d+\nu}} \lesssim \sum_{\ell=1}^{\mu}\left(\int_{\mathcal{Q}_{\widetilde{x}_{s}}^{d}(r)}\left|\phi_{\ell}\right|^{\rho}\right)^{\frac{4}{\rho(d+\nu)}}\left(\int_{\mathcal{Q}_{\widetilde{x}_{s}}^{d}(r)}\left|\nabla \phi_{\ell}\right|^{\rho}\right)^{\frac{2}{\rho}}
$$

and

$$
\rho=\frac{2 d(d+\nu+2)}{(d+2)(d+\nu)}>2 .
$$

An application of the Hölder inequality, gives that the r.h.s. of (A.2) is bounded as 


$$
\begin{aligned}
& \sum_{\ell=1}^{\mu}\left(\int_{\mathcal{Q}_{\tilde{x}_{s}}^{d}(\rho)}\left|\phi_{\ell}\right|^{\rho}\right)^{\frac{4}{\rho(d+\nu)}}\left(\int_{\mathcal{Q}_{\widetilde{x}_{s}}^{d}(r)}\left|\nabla \phi_{\ell}\right|^{\rho}\right)^{\frac{2}{\rho}} \\
\leqslant & C \sum_{\ell=1}^{\mu}\left(\int_{\mathcal{Q}_{\tilde{x}_{s}}^{d}(r)}\left|\phi_{\ell}\right|^{2}\right)^{\frac{2}{d+\nu}}\left(\int_{\mathcal{Q}_{\widetilde{x}_{s}}^{d}(r)}\left|\nabla \phi_{\ell}\right|^{2}\right) \\
\leqslant & C\left(\sum_{\ell=1}^{\mu}\left\|\phi_{\ell}\right\|_{L^{2}\left(\mathcal{Q}_{\tilde{x}_{s}}^{d}(r)\right)}\right)^{\frac{4}{d+\nu}} \sum_{\ell=1}^{\mu}\left\|\phi_{\ell}\right\|_{H^{1}\left(\mathcal{Q}_{\widetilde{x}_{s}}^{d}(r)\right)}^{2} .
\end{aligned}
$$

with $C>0$ a constant depending on meas $_{d}\left(\mathcal{Q}_{\widetilde{x}_{s}}^{d}(r)\right)$. From (A.2) and (A.3) one can get

$$
\|\phi\|_{L}^{\frac{2 d+2 \nu+4}{d+\nu}} \underset{\frac{2 d+2 \nu+4}{d+\nu}}{\left(\mathcal{Q}_{\widetilde{x}_{S}}^{d}(r)\right)^{\mu}} \leqslant\left(\|\phi\|_{L^{2}\left(\mathcal{Q}_{\tilde{x}_{S}}^{d}(r)\right)^{\mu}}\right)^{\frac{4}{d+\nu}}\|\phi\|_{H^{1}\left(\mathcal{Q}_{\widetilde{x}_{S}}^{d}(r)\right)^{\mu}}^{2} .
$$

Hence summing over $s$ we obtain

$$
\begin{aligned}
\|\phi\|_{L}^{\frac{2 d+2 \nu+4}{d+\nu}} \frac{\frac{2 d+2 \nu+4}{d+\nu}}{\left(\mathbb{R}^{d}\right)^{\mu}} & \leqslant\left(\sup _{s \in \mathbb{N}}\|\phi\|_{L^{2}\left(\mathcal{Q}_{\widetilde{x}_{s}}^{d}(r)\right)^{\mu}}\right)^{\frac{4}{d}} \sum_{s \in \mathbb{N}}\|\phi\|_{H^{1}\left(\mathcal{Q}_{\widetilde{x}_{s}}^{d}(r)\right)^{\mu}}^{2} \\
& \leqslant C\left(\sup _{x \in \mathbb{R}^{d}}\|\phi\|_{L^{2}\left(\mathcal{Q}_{\tilde{x}}^{d}(r)\right)^{\mu}}\right)^{\frac{4}{d+\nu}}\|\phi\|_{H^{1}\left(\mathbb{R}^{d}\right)^{\mu}}^{2},
\end{aligned}
$$

which is the estimate (A.1), with the constants involved independent from $s$ because the estimate above is translation invariant.

\section{REFERENCES}

[1] R. Adams, J. Fournier, Sobolev spaces, 2nd ed., Academic Press, 2003.

[2] A. Arora, Scattering of radial data in the focusing NLS and generalized Hartree equations, arXiv:1904.05800 [math.AP].

[3] A. Arora, S. Roudenko, Global behavior of solutions to the focusing generalized Hartree Equation, arXiv:1904.05339 [math.AP].

[4] N. Benedikter, V. Jaksic, M. Porta, C. Saffirio, B. Schlein, Mean-field Evolution of Fermionic Mixed States, Comm. Pure Appl. Math. 69 (2016), 2250-2303.

[5] N. Benedikter, J. Sok, and J. P. Solovej, The Dirac-Frenkel Principle for Reduced Density Matrices, and the Bogoliubov-de-Gennes Equations, Annales Henri Poincaré 19(4) (2018), 1167-1214.

[6] C. Bonanno, P. d'Avenia, M. Ghimenti, M. Squassina, Soliton dynamics for the generalized Choquard equation, J. Math. Anal. Appl., 417 (2014), 180-199.

[7] B. Cassano, M. Tarulli, $H^{1}$-scattering for Systems of $N$-defocusing Weakly Coupled NLS Equations in Low Space Dimensions. J. Math. Anal. Appl. 430 (2015) 528-548.

[8] R. Carles, W. Lucha, E. Moulay, Higher order Schrödinger and Hartree-Fock equations. Journal of Mathematical Physics, American Institute of Physics (AIP), 2015, 56 (12), pp.122-301. 
[9] T. Cazenave, Semilinear Schrödinger equations, Courant Lecture Notes in Mathematics, 10, New York University Courant Institute of Mathematical Sciences, New York, 2003.

[10] P. Choquard, J. Stubbe, The one-dimensional Schrödinger-Newton equations, Lett. Math. Phys. 81(2), (2007, 177-184)

[11] P. Choquard, J. Stubbe, M Vuffray, Stationary solutions of the SchrödingerNewton model-an ODE approach, Differ. Integral Equ. 21(7-8), (2008), 665679.

[12] A. Elgart, B. Schlein, Mean field dynamics of boson stars, Comm. Pure Appl. Math., 60(4), (2007), 500-545.

[13] B. Feng, X. Yuan. On the Cauchy problem for the Schrödinger-Hartree equation, Evolution Equations \& Control Theory, 2015, 4 (4) : 431-445. doi: 10.3934/eect.2015.4.431.

[14] V. A. Fock, Näherungsmethode zur lösung des quantenmechanischen mehrkörperproblems. Zeit. für Physik, 61(1-2), (1930), 126-148.

[15] J. Fröhlich, E. Lenzmann, Dynamical collapse of white dwarfs in Hartree-and Hartree-Fock theory, Commun. Math. Phys., 274, (2007), 737-750.

[16] H. Genev, G. Venkov, Soliton and blow-up solutions to the time-dependent Schrödinger-Hartree equation, Discrete Contin. Dyn. Syst. Ser. S, 5 (2012), 903-923.

[17] V. Georgiev, A. Stefanov, On the classification of the spectrally stable standing waves of the Hartree problem, Physica D: Nonlinear Phenomena, 370, (2018), 29-39.

[18] J. Ginibre, T. Ozawa, Long range scattering for nonlinear Schrdinger and Hartree equations in space dimension $n \geqslant 2$, Comm. Math. Phys., 151, (1993), 619-645.

[19] J. Ginibre, G. Velo, On a class of nonlinear Schrödinger equations with nonlocal interaction, Math. Z., 170(2), (1980), 109-136.

[20] J. Ginibre, G. Velo, Scattering theory in the energy space for a class of Hartree equations, Nonlinear wave equations, 263, (1998), 29-60.

[21] J. Ginibre, G. Velo. Quadratic morawetz inequalities and asymptotic completeness in the energy space for nonlinear Schrödinger and Hartree equations, Quart. Appl. Math. 68 (2010), 113-134 .

[22] E. Lenzmann, Well-posedness for semi-relativistic Hartree equations of critical type, Math. Phys. Anal. Geom., 10(1), (2007), 43-64,.

[23] N. Hayashi, T. Ozawa, Scattering theory in the weighted $L^{2}\left(\mathbb{R}^{n}\right)$ spaces for some Schrödinger-Hartree equations, Ann. Inst. Henri Poincar Phys. Théor. 48, 17-37.

[24] E. Lieb, Existence and uniqueness of the minimizing solution of Choquard's nonlinear equation, Studies in Appl. Math., 57 (1976), 93-105.

[25] M. Keel, T. Tao, Endpoint Strichartz estimates. 120, 5 (1998), 955-980.

[26] M. Lewin, N. Rougerie, Derivation of Pekar's polarons from a microscopic model of quantum crystal, SIAM J. Math. Anal., 45 (2013), 1267-1301.

[27] X. Li, Global existence and blowup for Choquard equations with an inversesquare potential, eprint arXiv:1902.04739.

[28] P. L. Lions, The concentration-compactness principle in the calculus of variations. The locally compact case. part 1 and partm2, Annales de l'institut Henri Poincaré (C) Analyse non lineaire, 1 no. 2 (1984), 09-145.

[29] C. Miao, G., Xu, L., Zhao, Global well-posedness and scattering for the energycritical, defocusing Hartree equation for radial data, Journal of Functional Analysis, 253, (2007), 605-627

[30] C. Miao, G., Xu, L., Zhao, The Cauchy problem of the Hartree equation, J. PDEs, 21, (2008), 22-44. 
[31] C. Miao, G., Xu, L., Zhao, On the blow-up phenomenon for the mass-critical focusing Hartree equation in $\mathbb{R}^{4}$, Colloq. Math., 119 (2010), 23-50.

[32] V. Moroz, J. V. Schaftingen, Groundstates of nonlinear Choquard equations: Existence, qualitative properties and decay asymptotics, J. Funct. Anal., 265 (2013), 153-184.

[33] K. Nakanishi, Energy scattering for nonlinear Klein-Gordon and Schrödinger equations in spatial dimensions 1 and 2. J. Fund. Anal. 169: 201-225, 1999.

[34] H. Nawa, T., Ozawa, Nonlinear scattering with nonlocal interaction. Comm. Math. Phys., 1992, 146, 259-275

[35] R. Penrose, Quantum computation, entanglement and state reduction, Phil. Trans. R. Soc., 356 (1998), 1927-1939.

[36] F. Planchon, L. Vega, Bilinear virial identities and applications, Ann. Sci. Éc. Norm. Supér. (4) 42 (2009), no. 2, 261-290.

[37] M. Tarulli, $H^{2}$-scattering for Systems of weakly Coupled fourthorder NLS equations in low Space dimensions, Potential Anal (2018). https://doi.org/10.1007/s11118-018-9712-8

[38] S. Terracini, N. Tzvetkov, N. Visciglia, The Nonlinear Schrödinger equation ground states on product spaces, 2012, Analysis \& PDE, to appear.

[39] N. Tzvetkov, N. Visciglia, Well-posedness and scattering for $N L S$ on $\mathbb{R}^{d} \times \mathbb{T}$ in the energy space, Rev. Mat. Iberoam. 32 (2016), no. 4, 1163-1188.

[40] N. Visciglia, On the decay of solutions to a class of defocusing, NLS. Math. Res. Lett. 16 (2009), no. 5, 919-926.

[41] T. Wada, Scattering theory for time-dependent Hartree-Fock type equation, Osaka J. Math., 36 (1999) 905-918.

Mirko Tarulli: Dipartimento di Matematica, Università Degli Studi di Pisa Largo Bruno Pontecorvo 5 I - 56127 Pisa. Italy. Faculty of Applied Mathematics and Informatics, Technical University of Sofia, Kliment Ohridski Blvd. 8, 1000 Sofia, and IMI BAS, Acad. Georgi Bonchev Str., Block 8, 1113 Sofia, BULGARIA

E-mail address: mta@tu-sofia.bg

George Venkov: Faculty of Applied Mathematics and Informatics, TechniCal University of Sofia, Kliment Ohridski Blvd. 8, 1000 Sofia, Bulgaria

E-mail address: gvenkov@tu-sofia.bg 